\title{
The Periodicity of the Accuracy of Numerical Integration Methods for the Solution of Different Engineering Problems
}

\author{
Toukir Ahmed Chowdhury, Towhedul Islam, Ahmad Abdullah Mujahid, and Md. Bayazid Ahmed
}

Department of Mechanical Engineering, Chittagong University of Engineering \& Technology, Chattogram-4349, Bangladesh

Received: October 05, 2021, Revised: November 30, 2021, Accepted: November 30, 2021, Available Online: December 23, 2021

\begin{abstract}
Newton-Cotes integration formulae have been researched for a long time, but the topic is still of interest since the correctness of the techniques has not yet been explicitly defined in a sequence for diverse engineering situations. The purpose of this paper is to give the readers an overview of the four numerical integration methods derived from Newton-Cotes formula, namely the Trapezoidal rule, Simpson's 1/3rd rule, Simpson's 3/8th rule, and Weddle's rule, as well as to demonstrate the periodicity of the most accurate methods for solving each engineering integral equation by varying the number of sub-divisions. The exact expressions by solving the numerical integral equations have been determined by Maple program and comparisons have been done using Python version 3.8.
\end{abstract}

Keywords: Numerical Integration Accuracy, Trapezoidal Rule, Simpson's 1/3 Rule, Simpson's 3/8 Rule, Weddle's Rule.

This work is licensed under a Creative Commons Attribution-Non Commercial 4.0 International License.

\section{Introduction}

Throughout the entire history of mathematical research, integration is undoubtedly one of the most important mathematical concepts ever conceived. An integral is a mathematical term that defines displacement, area, volume, and other ideas that result from the combination of infinitesimal data. The process of determining integrals is known as integration.

Numerical integration is the process of estimating the value of a definite integral from the estimated numerical values of the integrand. If the numerical integration is performed on a single variable, it is called Quadrature, while for multiple variables, it is called Cubature. Scientists and engineers mostly utilize numerical integration to get an approximate solution for definite integrals that cannot be solved analytically. The reasons for which numerical integration is preferred over analytical are:

- Although there is a closed form solution, calculating the answer numerically can be difficult.

- The integrand $f(x)$ may only be known at a few locations, as determined through sampling.

- Many integrals are not analytically evaluable or have no closed form solution. e.g. $\int_{0}^{t} e^{-2 x^{3}} d x$

- Although the integrand $\mathrm{f}(\mathrm{x})$ is not explicitly known, a collection of data points for this integrand is provided.

The term 'Numerical Integration' was first coined in 1915 in the booklet named A Course in Interpolation and Numeric Integration for the Mathematical Laboratory by David Gibb.

Many academic areas, including applied mathematics, geometry, finance, statistics, economics, and engineering, use numerical integration methods. The available numerical integration methods include Quadrature methods, Gaussian integration, Monte-Carlo integration, Adaptive Quadrature, and the Euler-Maclaurin formula, which are used to compute complex functions. The Newton-Cotes formulas are also acknowledged as the Newton-Cotes quadrature standards or truly Newton-Cotes laws. These are the numerical integration implementation techniques (also regarded as quadrature), especially focused on measuring the integrand at equally spaced numerical analysis factors. The methods are named after Isaac Newton and Roger Cotes. There are two forms of the method for Newton-Cotes; Open Newton-Cotes and Closed Newton-Cotes. Trapezoidal rule, Simpson 1/3 rule, Simpson 3/8 rule, Weddle's rule and Boole's rule originate from the closed Newton-Cotes formula. On the other hand, Midpoint law, Trapezoid process, Milne's rule is derived from the formula of open Newton-Cotes.

The different numerical integration equations are covered in works by S.S. Sastry [1]-[2], R.L. Burden [3]-[4], J.H. Mathews [5]-[6], and others. M. Concepcion Austin [4] was helpful in evaluating different numerical integration producers and addressing more sophisticated numerical integration techniques. Gordon K. Smith [5] made contributions to this discipline through his analytic study of numerical integration and a collection of 33 papers and books on the subject. Rajesh Kumar Sinha [6] attempted to evaluate an integrable polynomial without using the Taylor Series. Gerry Sozio [7] examined a comprehensive overview of different numerical integration methods. J.Oliver [8] explored the multiple evaluation procedures of definite integrals using higher-order formulas. A. Nataranjan and N. Mohankumar [9] compared several quadrature methods for approximating Cauchy principal value integrals. D.J. Liu, J.M. Wu, and D.H. Yu [10] explored the super convergence of the Newton-Cotes rule for Cauchy principal value integrals. Romesh Kumar Muthumalai [11] attempted to calculate the inaccuracy of numerical integration and differentiation, and he also developed several formulas for numerical differentiation by divided difference. Md. Mamun-UrRashid Khan [12] devised a novel technique to numerical integration strategies for uneven data space.

In the realm of applied mathematics, numerical integration has a wide range of applications, particularly in mathematical physics and computational chemistry [13]. It is also employed in population estimation [14], medical picture reconstruction [15], and physics [16]. Chapra SC showed in Applied Numerical Methods with MATLAB [17] the application of numerical methods to solve problems in engineering and science. 
Previously, many mathematical softwares like Mathcad [18], Matlab [19], Fortran, C, C++ [20]-[21], and Mathematica [22] have been used to solve integrals numerically. Caligaris et.al [22] had designed a tool which performs numerical integration in Mathematica by four methods namely Trapezoidal, Simpson's 1/3, Simpson's 3/8, and Gauss Quadrature with various points. In this paper, the use of numerical integration in different engineering applications has been focused on. Instead of using the other previously used languages, we used Python 3.8 to solve different integral equations. From the graphs and the values of individual problems, it is seen that with the change of interval, the most accurate method changes and there is an interesting pattern of accuracy that is followed in each engineering problem.

\section{Mathematical Model}

\subsection{Newton-Cotes formula}

Newton's forward difference formula for equally spaced intervals is given by

$$
\mathrm{y}(\mathrm{x})=\mathrm{y}_{0}+\mathrm{u} \Delta \mathrm{y}_{0}+\frac{u(u-1)}{2 !} \Delta^{2} \mathrm{y}_{0}+\frac{u(u-1)(u-2)}{3 !} \Delta^{3} \mathrm{y}_{0}+\ldots \ldots
$$

Here, $u=\frac{x-x_{0}}{h}, x=x_{0}+u h, d x=h d u$

$\int_{x_{0}}^{x_{n}} y d x$

$=\int_{x_{0}}^{x_{0}+n h} f(x) d x$

$=\int_{0}^{n}\left(\mathrm{y}_{0}+\mathrm{u} \Delta \mathrm{y}_{0}+\frac{u(u-1)}{2 !} \Delta^{2} \mathrm{y}_{0}+\frac{u(u-1)(u-2)}{3 !} \Delta^{3} \mathrm{y}_{0}+\ldots \ldots\right)$

$h d u$

$=h \int_{0}^{n}\left(\mathrm{y}_{0}+\mathrm{u} \Delta \mathrm{y}_{0}+\frac{u(u-1)}{2 !} \Delta^{2} \mathrm{y}_{0}+\frac{u(u-1)(u-2)}{3 !} \Delta^{3} \mathrm{y}_{0}+\ldots \ldots\right)$

$d u$

$=h\left[\mathrm{ny}_{0}+\frac{n^{2}}{2} \Delta \mathrm{y}_{0}+\frac{1}{2}\left(\frac{n^{3}}{3}-\frac{n^{2}}{2}\right) \Delta^{2} \mathrm{y}_{0}+\ldots \ldots \ldots\right]$

This is the required Newton-Cotes Quadrature formula.

By putting $n=1$ in the Quadrature formula, we get the Trapezoidal rule,

$$
\int_{x_{0}}^{x_{n}} y d x=\frac{h}{2}\left[\left(\mathrm{y}_{0}+\mathrm{y}_{\mathrm{n}}\right)+2\left(\mathrm{y}_{1}+\mathrm{y}_{2}+\mathrm{y}_{3}+\ldots \ldots .+\mathrm{y}_{\mathrm{n}-1}\right)\right]
$$

By putting $\mathrm{n}=2$ in the Quadrature formula, we get the Simpson's $\frac{1}{3}$ rule,

$$
\begin{aligned}
\int_{x_{0}}^{x_{n}} y d x=\frac{h}{3}\left[\left(\mathrm{y}_{0}+\mathrm{y}_{\mathrm{n}}\right)+\right. & 2\left(\mathrm{y}_{2}+\mathrm{y}_{4}+\ldots .+\mathrm{y}_{2 \mathrm{n}}\right)+4\left(\mathrm{y}_{1}+\mathrm{y}_{3}\right. \\
& +\ldots)]
\end{aligned}
$$

By putting $\mathrm{n}=3$ in the Quadrature formula, we get the Simpson's $\frac{3}{8}$ rule,

$$
\begin{gathered}
\int_{x_{0}}^{x_{n}} y d x=\frac{3 h}{8}\left[\left(\mathrm{y}_{0}+\mathrm{y}_{\mathrm{n}}\right)+3\left(\mathrm{y}_{1}+\mathrm{y}_{2}+\mathrm{y}_{4}+\mathrm{y}_{5}+\ldots\right)+2\left(\mathrm{y}_{3}\right.\right. \\
\left.\left.+\mathrm{y}_{6}+\ldots\right)\right]
\end{gathered}
$$

By putting $n=6$ in the Quadrature formula, we get the Weddle's rule,

$$
\begin{array}{r}
\int_{x_{0}}^{x_{n}} y d x=\frac{3 h}{10}\left[\left(\mathrm{y}_{0}+\mathrm{y}_{\mathrm{n}}\right)+5\left(\mathrm{y}_{1}+\mathrm{y}_{7}+\mathrm{y}_{13}+\ldots \ldots\right)+\left(\mathrm{y}_{2}+\right.\right. \\
\left.\mathrm{y}_{8}+\mathrm{y}_{14}+\ldots \ldots\right)+6\left(\mathrm{y}_{3}+\mathrm{y}_{9+} \mathrm{y}_{15}+\ldots . .\right)+\left(\mathrm{y}_{4}+\mathrm{y}_{10}+\mathrm{y}_{16}\right. \\
+\ldots \ldots)+5\left(\mathrm{y}_{5}+\mathrm{y}_{11}+\mathrm{y}_{17}+\ldots \ldots\right)
\end{array}
$$

\section{Different Engineering Problems \& Their Applications}

\subsection{Spring}

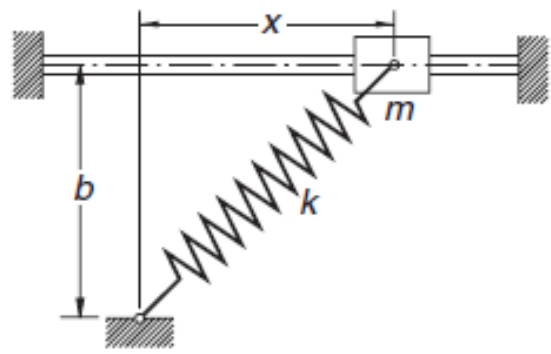

Fig. 1 Spring [23].

"The mass $m$ is attached to a spring (see ) of free length $b$ and stiffness $k$. The coefficient of friction between the mass and the horizontal rod is $\mu$. The acceleration of the mass can be shown to be $a=-f(x)$, where

$$
f(x)=\mu g+\frac{k}{m}(\mu b+x)\left(1-\frac{b}{\sqrt{b^{2}+x^{2}}}\right)
$$

If the mass is released from rest at $x=b$, its speed at $x=0$ is given by

$$
v_{0}=\sqrt{2 \int_{0}^{b} f(x) d x}
$$

Compute $v_{0}$ by using the data $m=0.8 \mathrm{~kg}, b=0.4 \mathrm{~m}, \mu=0.3$, $k=80 \mathrm{~N} / \mathrm{m}$ and $g=9.81 \mathrm{~m} / \mathrm{s}^{2}$ [23]."

\subsubsection{Actual Solution}

When $x=0$, the speed of the mass $m$ will be

$$
\begin{aligned}
v_{0} & =\sqrt{2 \int_{0}^{0.4} f(x) d x} \\
& =\sqrt{2 \int_{0}^{0.4} \mu g+\frac{k}{m}(\mu b+x)\left(1-\frac{b}{\sqrt{b^{2}+x^{2}}}\right) d x}
\end{aligned}
$$

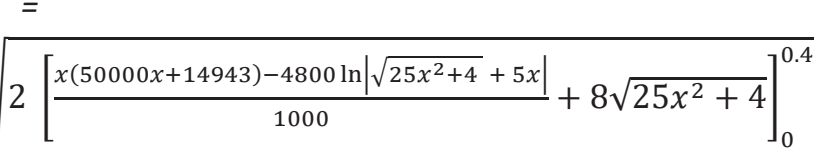

$$
=2.49767483245384 \mathrm{~m} / \mathrm{s}
$$

\subsubsection{Engineering Application}

The described problem and its solution can be used for controlling forces and movement in brakes and clutch systems, for minimizing shocks and vibrations in the suspension system of a car, energy conservation in the case of toys and watches, etc.

\subsection{Capacitor}

"A capacitor in an electrical circuit is initially at zero charge. At time $t$ of $1 \mathrm{~s}$, a switch is closed, and a time-dependent electric current $I(t)$ charges up the capacitor. The current is given as

$$
I(t)=4\left(1-e^{-0.5}\right) e^{-0.5(t-1)}\left(1-e^{-t}\right) .
$$
[24].' 


$$
\begin{aligned}
& \text { 3.2.1 Actual Solution } \\
& \begin{aligned}
Q(t) & =\int_{1}^{20} I(t) d t \\
= & \int_{1}^{20} 4\left(1-e^{-0.5}\right) e^{-0.5(t-1)}\left(1-e^{-t}\right) d t \\
= & {\left[-\frac{8(\sqrt{e}-1) e^{-1.5 t}\left(3 e^{t}-1\right)}{3}\right]_{1}^{20} } \\
= & 2.76152102437006 \text { coulomb }
\end{aligned}
\end{aligned}
$$

\subsubsection{Engineering Application}

The above capacitor equation and its solutions can be used in both AC and DC capacitors, to reduce ripple voltage in filter circuits, in a single phase motor, for filtering and tuning circuits, as a storage in digital equipment and long-time constant circuits, for frequency selection in tuning circuits, energy storage in a video flash circuits, suppresses radio frequency interference as used in a snubber circuit etc.

\subsection{Volume Flow Rate of Turbulent Flow}

"The velocity profile in the turbulent flow of a fluid in a smooth circular pipe may be represented by the empirical powerlaw equation

$$
U(x)=5\left(1-\frac{x}{R}\right)^{1 / 7}
$$

Where $U(x)$ is the axial velocity in the pipe, in $\mathrm{m} / \mathrm{s}, x$ is the radial distance from the axis, in meters, and $R$ is the radius of the pipe. The total volume flow rate in the pipe is then given by the integral $\int_{0}^{R} U(x) 2 \pi x d x$. Compute the total volume flow rate if $R=0.5 \mathrm{~m} \mathrm{[24]."}$

\subsubsection{Actual Solution}

The total volume flow rate,

$\dot{V}=\int_{0}^{.5} U(x) 2 \pi x d x$

$$
\begin{aligned}
& =\int_{0}^{0.5} 5\left(1-\frac{x}{0.5}\right)^{1 / 7} 2 \pi x d x \\
& =\left[-10 \pi\left(\frac{7}{32}(1-2 x)^{8 / 7}-\frac{7}{60}(1-2 x)^{15 / 7}\right)\right]_{0}^{0.5}
\end{aligned}
$$

$$
=3.20704250053958 \mathrm{~m}^{3} / \mathrm{s}
$$

\subsubsection{Engineering Application}

The equation of volume flow rate of turbulent flow is used for the external flow of air and water over all vehicles types, including automobiles, helicopters, bridges, and submarines, dimples in the golf ball, flow near an airplane, in many manufacturing devices, and machinery like tanks, ducts, precipitators, gas scrubbers, complex scraped surface heat exchangers, etc.

\subsection{Wind Force}

The pressure $p$ on a $10 \mathrm{~m}$ high structure due to the wind is given by the expression

$$
p(x)=\frac{150 x}{1+e^{x}}
$$

where $x$ is measured in meters from the bottom of the structure and the pressure is in $\mathrm{N} / \mathrm{m}^{2}$. If the structure is $2 \mathrm{~m}$ wide, the total force due to wind is given by the integral

$$
\int_{0}^{10} 2 p(x) d x
$$

Compute the total force due to wind. [24]

\subsubsection{Actual Solution}

The total force on the structure due to wind $F$ is given by the equation

$$
\begin{aligned}
F & =\int_{0}^{10} 2\left(\frac{150 x}{1+e^{x}}\right) d x \\
& =\left[-300 x \ln \left(e^{x}+1\right)-300 \operatorname{Li}_{2}\left(-e^{x}\right)+150 x^{2}\right]_{0}^{10} \\
& =246.590293505238 \mathrm{~N}
\end{aligned}
$$

\subsubsection{Engineering Application}

The equation of wind force can be used in the field of electricity generation by the windmills, sailing vessels, sailboard, grain milling for sports like windsurfing, land surfing, kite surfing, etc.

\subsection{Total Momentum Flow}

"The velocity profile in the turbulent flow of a fluid in a smooth circular pipe may be represented by the empirical powerlaw equation

$$
U(x)=5\left(1-\frac{x}{R}\right)^{1 / 7}
$$

where $U(x)$ is the axial velocity in the pipe, in $\mathrm{m} / \mathrm{s}, x$ is the radial distance from the axis, in meters, and $R$ is the radius of the pipe. The momentum flow is given by the integral $\int_{0}^{R} 2 \pi x \rho[U(x)]^{2} d x$, where $\rho$ is the fluid density, given as 1 $\mathrm{kg} / \mathrm{m}^{3}$ for the fluid considered. Compute the total momentum flow if $R=0.5 \mathrm{~m}$ [24]."

\subsubsection{Actual Solution}

Total momentum flow $M$ is given by the equation

$$
\begin{aligned}
M & =\int_{0}^{0.5} 2 \pi x \rho[U(x)]^{2} d x \\
& =\int_{0}^{0.5} 2 \pi x(1)\left[5\left(1-\frac{x}{0.5}\right)^{1 / 7}\right]^{2} d x \\
& =\left[-\frac{175 \pi(18 x+7)(1-2 x)^{9 / 7}}{288}\right]_{0}^{0.5} \\
& =13.3626770855815 \mathrm{~kg} . \mathrm{m} / \mathrm{s}
\end{aligned}
$$

\subsubsection{Engineering Application}

The momentum flow equation is generally used for nonuniform flow through a suddenly enlarged pipe, jet propulsion, propellers, hydraulic jump in an open channel, etc.

\subsection{Water Force on a Vertical Plate}

The force $F(x)$ exerted per centimeter on a vertical plate immersed in flowing water is given by the expression

$$
F(x)=1.5 x^{3} e^{-x}
$$

where $x$ is measured from the top of the plate and $F(x)$ is in $\mathrm{N} / \mathrm{cm}$. If the plate is $10 \mathrm{~cm}$ high, compute the total force $F_{T}$, in Newtons, on the plate is given by [24]

$$
F_{T}=\int_{0}^{10} F(x) d x
$$




\subsubsection{Actual Solution}

The total force on the plate is given by the equation

$$
\begin{aligned}
F_{T} & =\int_{0}^{10} F(x) d x \\
& =\int_{0}^{10} 1.5 x^{3} e^{-x} d x \\
& =\left[-1.5 e^{-x}\left(x^{3}+3 x^{2}+6 x+6\right)\right]_{0}^{10} \\
& =8.90697554391667 \mathrm{~N}
\end{aligned}
$$

\subsubsection{Engineering Application}

The equation of water force on a vertical plate is used in dam constructions, syringe construction, water tank construction, submarine construction, safety equipment for sea drivers, etc.

\subsection{Surface Tension}

"The meniscus of a liquid film supported by surface tension can often be represented as

$$
h(x)=A e^{-a^{2} x^{2}}
$$

where $h(x)$ is the height as a function of horizontal distance $x$ and $A$ and $a$ are constants. The total volume of liquid supported by surface tension is then given by the integral $W=\int_{0}^{L} h(x) d x$, where $W$ is the width of the meniscus and $L$ is its length. If $W, L$, $h$, and $x$ are all in centimeters, compute this volume for $A=0.8$, $a=2.0, W=1 \mathrm{~cm}$ and $L=1 \mathrm{~cm}$." [24].

\subsubsection{Actual Solution}

The total volume of liquid supported by surface tension is $W=\int_{0}^{L} h(x) d x=\int_{0}^{1} A e^{-a^{2} x^{2}} d x=\int_{0}^{1} 0.8 e^{-2^{2} x^{2}} d x$

$$
\begin{aligned}
& =\left[\frac{\sqrt{\pi}}{5} \operatorname{erf}(2 \mathrm{x})\right]_{0}^{1} \\
& =0.35283255630497 \mathrm{~cm}^{3}
\end{aligned}
$$

\subsubsection{Engineering Application}

The above equation of surface tension is used to remove pollutants from water and air, inkjet printer, scientific understanding of capillarity, wetting or beading a liquid on a surface, etc.

\subsection{Force on Sailboat Mast}

"The force on a sailboat mast can be represented by the following function:

$$
F=\int_{0}^{H} 200\left(\frac{Z}{5+Z}\right) e^{-2 z / H} d z
$$

where $z=$ the elevation above the deck and $H=$ the height of the mast. Compute $F$ for the case where $H=30$." [25]

\subsubsection{Actual Solution}

Force on the sailboat mast is given by the equation

$$
\begin{aligned}
F & =\int_{0}^{30} 200\left(\frac{Z}{5+Z}\right) e^{-2 z / 30} d z \\
& =\left[-3000 e^{-\frac{x}{15}}+1000 e^{\frac{1}{3}} E i_{1}\left(\frac{x+5}{15}\right)\right]_{0}^{30} \\
& =1480.5684800859 \mathrm{~N}
\end{aligned}
$$

\subsubsection{Engineering Application}

The equation of force on a sailboat mast is used for iceboats, sail-powered land vehicles, sailing ships, windsurfers, windmill sails, wind turbines, etc.

\subsection{Mass Flow Rate}

"The amount of mass transported via a pipe over a period of time can be computed as

$$
M=\int_{t_{1}}^{t_{2}} Q(t) c(t) d t
$$

where $M=$ mass (mg), $t_{1}=$ the initial time $(\min ), t_{2}=$ the final time $(\mathrm{min}), Q(t)=$ flow rate $\left(\mathrm{m}^{3} / \mathrm{min}\right)$, and $c(t)=$ concentration $\left(\mathrm{mg} / \mathrm{m}^{3}\right)$. The following functional representations define the temporal variations in flow and concentration:

$$
Q(t)=9+5 \cos ^{2}(0.4 t) ; c(t)=5 e^{-0.5 t}+2 e^{0.15 t},
$$

determine the mass transported between $t_{1}=2 \min$ and $t_{2}=$ $8 \min [25] . "$

\subsubsection{Actual Solution}

The amount of mass transported via pipe between 2 min and $8 \mathrm{~min}$ is

$$
\begin{aligned}
& M=\int_{2}^{8} Q(t) c(t) d t=\int_{2}^{8}\left(9+5 \cos ^{2}(0.4 t)\right)\left(5 e^{-0.5 t}+\right. \\
& \left.2 e^{0.15 t}\right) d t \\
& =\left[\frac { e ^ { - t / 2 } } { 1 4 1 5 1 } \left(e ^ { 1 3 t / 2 0 } \left(85440 \sin \frac{4 t}{5}+16020 \cos \frac{4 t}{5}+\right.\right.\right. \\
& \left.\left.2169820)+159000 \sin \frac{4 t}{5}-99375 \cos \frac{4 t}{5}-1627365\right)\right]_{2}^{8} \\
& =335.962530061625 \mathrm{~kg}
\end{aligned}
$$

\subsubsection{Engineering Application}

The mass flow rate equation is used in an industrial cooling tower, chemical component separation in distillation columns, liquid-liquid extraction, as an absorber such as active carbon beds, scrubbers or stripping, etc.

\subsection{Rocket Height}

"The upward velocity of a rocket can be computed by the following formula:

$$
v=u \ln \left(\frac{m_{0}}{m_{0}-q t}\right)-g t
$$

where $v=$ upward velocity, $u=$ velocity at which fuel is expelled relative to the rocket, $m_{0}=$ initial mass of the rocket at time $t=0, q=$ fuel consumption rate, and $g=$ downward acceleration of gravity (assumed constant $=9.81 \mathrm{~m} / \mathrm{s}^{2}$ ). If $u=$ $1850 \mathrm{~m} / \mathrm{s}, m_{0}=160,000 \mathrm{~kg}$, and $q=2500 \mathrm{~kg} / \mathrm{s}$, determine how high the rocket will fly in $30 \mathrm{~s}$ [25]."

\subsubsection{Actual Solution}

In $30 \mathrm{~s}$, the height of the rocket from the ground will be

$$
\begin{aligned}
& H=\int_{0}^{30} v d t=\int_{0}^{30}\left(1850 \ln \left(\frac{160000}{160000-2500 t}\right)-9.81 t\right) d t \\
&=\left[-4.905 t^{2}+1850(t-64) \ln \left(\frac{-64}{t-64}\right)+1850 t-\right. \\
&118400]_{0}^{30}=11299.8310550331 \mathrm{~m}
\end{aligned}
$$




\subsubsection{Engineering Application}

The described problem and its solution is used for measuring the rocket height from the surface. Although the rocket ejects fuel to provide thrust, it is an example of conservation of momentum where the system's mass is not constant. The rocket equation calculates the difference of velocity obtained by a rocket after burning an abundance of fuel that reduces the rocket's overall mass.

\subsection{Paratroopers}

"An armed paratrooper with ammunition weighing 322 pounds jumps from a plane with zero initial velocity. The troopers encounter negligible side wind in their descent. However, they encounter air resistance whose magnitude is 15 times the square of the descent velocity $v(t)$; that is, $15[v(t)]^{2}$. Assume that the mass of the parachute is negligible. Estimate the distance paratrooper will travel after $15 \mathrm{~s}$ [26]."

\subsubsection{Actual Solution}

The total mass of the falling body $m=322 / 32.2=10$ slugs, and the air resistance $R(t)=15[v(t)]^{2}$ as given. The instantaneous descending velocity $v(t)$ can be obtained by using

$$
\begin{gathered}
\frac{d v(t)}{d t}+\frac{R(t)}{m}=g \\
\gg \frac{d v(t)}{d t}+\frac{15[v(t)]^{2}}{10}=32.2
\end{gathered}
$$

Consequently, one may express the solution of the above equation with $v=v(t)$ as $v(t)=\frac{4.634\left(e^{13.9 t}-1\right)}{e^{13.9 t}-1}$

So, the distance paratroopers will travel is given by the equation,

$$
\begin{aligned}
& X=\int_{0}^{15} \frac{4.634\left(e^{13.9 t}-1\right)}{e^{13.9 t}+1} d t \\
& =\left[\frac{2317 \ln \left(e^{13.9 t}+1\right)}{3475}+\frac{2317 t}{500}\right]_{0}^{15}
\end{aligned}
$$

$$
=69.0478353906885 \mathrm{ft}
$$

\subsubsection{Engineering Application}

The stated equation and its solution are employed in military force distribution and transportation are frequently utilized in surprise attacks to take important targets such as airfields or bridges, to build an airhead for landing further units, like in the Battle of Crete, and so on.

\subsection{Horizontal Deflection}

"The part shown is formed from a $\frac{1}{8}$ in diameter steel wire, with $R=5$ in and $l=4 \mathrm{in}$. A force is applied with $P=1 \mathrm{lbf}$. Use Castigliano's method to estimate the horizontal deflection at point $A$ [27]."

\subsubsection{Actual Solution}

For the straight portion,

Momentum, $M_{A B}=\mathrm{Px} ; \quad \frac{\partial M_{A B}}{\partial P}=\mathrm{x}$

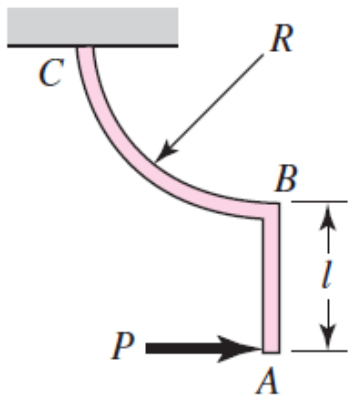

Fig. 2 Beam [27].

For the curved portion,

Momentum, $M_{B C}=\mathrm{P}[R(1-\cos \theta)+l]$;

$\frac{\partial M_{B C}}{\partial P}=[R(1-\cos \theta)+l]$

The horizontal deflection at point $A$ can be written as

$$
\begin{aligned}
& \delta=\int_{0}^{l} \frac{1}{E l}\left(M_{A B} \frac{\partial M_{A B}}{\partial P}\right) d x+\int_{0}^{\pi / 2} \frac{1}{E l}\left(M_{B C} \frac{\partial M_{B C}}{\partial P}\right) R d \theta \\
&=\frac{P l^{3}}{3 E l}+\frac{P R}{E l} \int_{0}^{\pi / 2}\left[R^{2}\left(1-2 \cos \theta+\cos ^{2} \theta\right)+2 R l(1-\right. \\
&\left.\cos \theta)+l^{2}\right] d \theta
\end{aligned}
$$

$$
\begin{gathered}
=\frac{(1)\left(4^{3}\right)}{3(30)\left(10^{6}\right) \pi\left(0.125^{4}\right) / 64}+\frac{(1)(5)}{(30)\left(10^{6}\right) \pi\left(0.125^{4}\right) / 64} \int_{0}^{\pi / 2}\left[5^{2}(1-\right. \\
\left.\left.2 \cos \theta+\cos ^{2} \theta\right)+2(5)(4)(1-\cos \theta)+4^{2}\right] d \theta
\end{gathered}
$$

$$
\begin{aligned}
& =\frac{(1)\left(4^{3}\right)}{3(30)\left(10^{6}\right) \pi\left(0.125^{4}\right) / 64}+\frac{(1)(5)}{(30)\left(10^{6}\right) \pi\left(0.125^{4}\right) / 64} \\
& {\left[\frac{25 \sin (2 \theta)-360 \sin \theta+374 \theta}{4}\right]_{0}^{\pi / 2}}
\end{aligned}
$$

$$
=0.85023052811512 \mathrm{in}
$$

\subsubsection{Engineering Application}

The horizontal deflection equation can be used in various places, including houses and bridges, lifting beams, gantry cranes, to prevent differential settlement and distribute lateral forces caused by earthquakes, strength calculations, etc.

\subsection{Block-Type Hand Brake (Moment of Frictional Forces)}

"The block-type hand brake shown in the Fig. 3 has a face width of 1.25 in and a mean coefficient of friction of 0.25 . For a maximum pressure on the shoe of 27.4 psi, find the moment of frictional forces [27]."

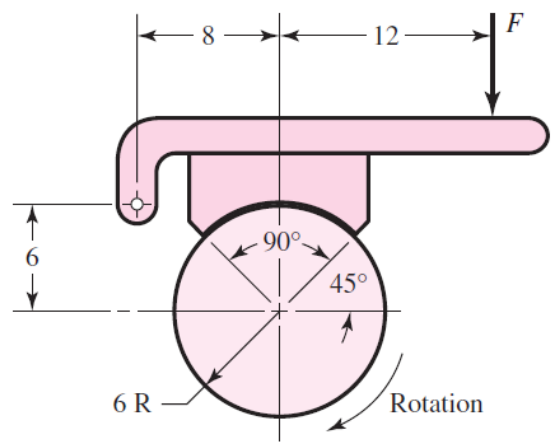

Fig. 3 Block type hand brake [27]. 


\subsubsection{Actual Solution}

Given, face width $b=1.25$ in, mean coefficient of friction $f$ $=0.25$, radius $r=6$ in, maximum pressure on the shoe $P_{a}=27.4$ psi.

Here, $\theta_{1}=45^{\circ}-\tan ^{-1}(6 / 8)=8.13^{\circ}, \theta_{2}=98.13^{\circ}, \theta_{a}=90^{\circ}$, $\mathrm{a}=\left(6^{2}+8^{2}\right)^{0.5}=10$ in

So the moment of friction forces can be written as

$$
\begin{aligned}
M_{f} & =\frac{f P_{a} b r}{\sin \theta_{a}} \int_{\theta_{1}}^{\theta_{2}} \sin \theta(r-a \cos \theta) d \theta \\
& =\frac{(0.25)(27.4)(1.25)(6)}{1} \int_{8.13^{\circ}}^{98.13^{\circ}} \sin \theta(6-10 \cos \theta) d \theta \\
& =\frac{(0.25)(27.4)(1.25)(6)}{1}[\cos \theta(5 \cos \theta-6)]_{8.13^{\circ}}^{98.13^{\circ}} \\
& =102.144340253044 \mathrm{lbf} . \mathrm{in}
\end{aligned}
$$

\subsection{Block-Type Hand Brake (Moment of Normal Forces)}

"The block-type hand brake shown in Fig. 4 has a face width of 1.25 in and a mean coefficient of friction of 0.25 . For a maximum pressure on the shoe of $27.4 \mathrm{psi}$, find the moment of normal forces [27]."

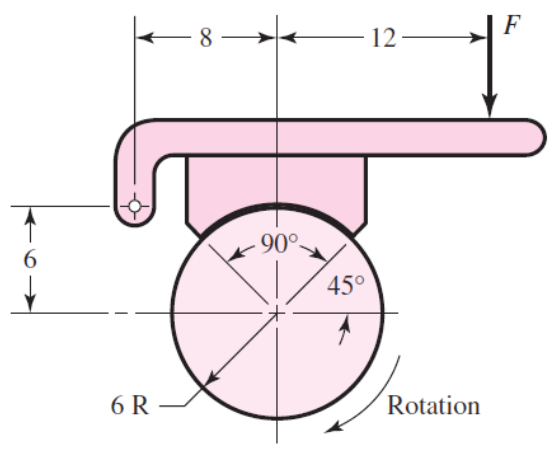

Fig. 4 Block type hand brake [27].

\subsubsection{Actual Solution}

Given, face width $b=1.25$ in, mean coefficient of friction $f$ $=0.25$, radius $r=6 \mathrm{in}$, maximum pressure on the shoe $P_{a}=27.4$ psi.

Here, $\theta_{1}=45^{\circ}-\tan ^{-1}(6 / 8)=8.13^{\circ}, \theta_{2}=98.13^{\circ}, \theta_{a}=90^{\circ}$, $\mathrm{a}=\left(6^{2}+8^{2}\right)^{0.5}=10$ in

So the moment of friction forces can be written as $M_{N}=\frac{a P_{a} b r}{\sin \theta_{a}} \int_{\theta_{1}}^{\theta_{2}} \sin ^{2} \theta d \theta=\frac{(10)(27.4)(1.25)(6)}{1} \int_{8.13^{\circ}}^{98.13^{\circ}} \sin ^{2} \theta d \theta$

$$
=\frac{(10)(27.4)(1.25)(6)}{1}\left[\frac{2 \theta-\sin (2 \theta)}{4}\right]_{8.13^{\circ}}^{98.13^{\circ}}
$$

$$
=1901.68970153642 \text { lbf.in }
$$

\subsubsection{Engineering Application}

The above equation of hand brake is used in the automobile. Hand brakes are sometimes referred to as parking brakes, and emergency brakes are used to hold the vehicle steady. It can operate in various places, including racing cars.

\subsection{Speed of Block}

"The block of mass $M=2 \mathrm{~kg}$ is subjected to a force having a constant direction and a magnitude $F=k /(a+b x)$ where, $k=$ $300 \mathrm{~N}, a=1, b=1 \mathrm{~m}^{-1}$. When $x=x_{1}=4 \mathrm{~m}$, the block is moving to the left with a speed $v_{1}=8 \mathrm{~m} / \mathrm{s}$. Determine its speed when $x$ $=x_{2}=12 \mathrm{~m}$. The coefficient of kinetic friction between the block and the ground is $\mu_{k}=0.25$ [28].'

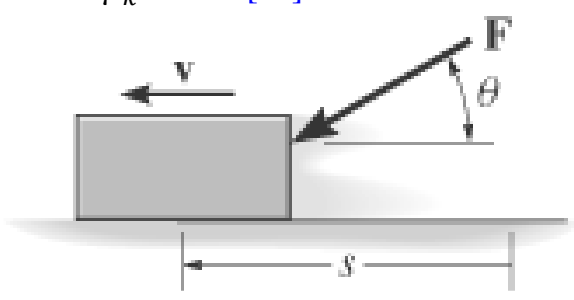

Fig. 5 Block [28].

\subsubsection{Actual Solution}

Considering $\theta=30^{\circ}$ and $\mathrm{g}=9.81 \mathrm{~ms}^{-2}$, the speed of the block when $x=x_{2}=12 \mathrm{~m}$ will be written as

$$
\begin{aligned}
& v=\sqrt{v_{1}^{2}+\frac{2\left(\int_{x_{1}}^{x_{2}} \frac{k \cos \theta}{a+b x} d x-\mu_{k} \int_{x_{1}}^{x_{2}} M g+\frac{k \sin \theta}{a+b x} d x\right)}{M}} \\
& =\sqrt{8^{2}+\frac{2\left(300 \int_{4}^{12} \frac{\cos 30^{\circ}}{1+x} d x-0.25 \int_{4}^{12} 2(9.8)+\frac{300 \sin 30^{\circ}}{1+x} d x\right)}{2}} \\
& =15.4005674022625 \mathrm{~m} / \mathrm{s}
\end{aligned}
$$

\subsubsection{Engineering Application}

The problem mentioned can be utilized to measure the speed when friction is used. We can walk due to friction; friction is used to hold together other items, heat switching through matches, and transportation system also requires acceleration friction, braking, etc.

\subsection{Rocket Velocity}

"A rocket has an empty weight $W_{1}=500 \mathrm{lb}$ and carries fuel of weight $W_{2}=300 \mathrm{lb}$. If the fuel is burned at the rate $c=15$ $l b / s$ and ejected with a relative velocity $v_{D R}=4400 \mathrm{ft} / \mathrm{s}$ determine the maximum speed attained by the rocket starting from rest. Neglect the effect of gravitation on the rocket. (Gravitational acceleration $g=32.2 \mathrm{ft} / \mathrm{s}$ ) [28]"

\subsubsection{Actual Solution}

Initial mass of the rocket $m_{0}=\frac{W_{1}+W_{2}}{\mathrm{~g}}=\frac{800}{32.2} \mathrm{lb}$

The maximum speed occurs when all the fuel is consumed, that is, where time $t=\frac{W_{2}}{c}=\frac{300}{15}=20 \mathrm{~s}$

The maximum speed of the rocket,

$v_{\text {max }}=\int_{0}^{t} \frac{\frac{c}{\mathrm{~g}} v_{D R}}{m_{0}-\frac{c}{\mathrm{~g}} t} d t=\int_{0}^{20} \frac{\frac{15}{32.2}(4400)}{\frac{800}{32.2}-\frac{15}{32.2} t} d t$

$=[-4400 \ln |3 t-160|]_{0}^{20}$

\section{$=2068.01596868123 \mathrm{ft} / \mathrm{s}$}

\subsubsection{Engineering Application}

Rockets are used to launch artificial satellites, human spaceflight, and space exploration, as well as for pyrotechnics, weapons, and ejection seats. Chemical rockets are the most frequent type of high-powered rocket, and they typically generate a high-speed exhaust by mixing fuel and an oxidizer. 


\subsection{Gear Box}

"Morse Industrial manufactures the speed reducer shown. If a motor drives the gear shaft $S$ with an angular acceleration $\alpha=$ $k e^{b t}$, where $k=0.4 \mathrm{rad} / \mathrm{s}^{2}, b=1 \mathrm{~s}^{-1}$. Determine the angular velocity of shaft $E$ at time $t=2 \mathrm{~s}$ after starting from rest. The radii of gear A, B, C, and D are $20 \mathrm{~mm}, 80 \mathrm{~mm}, 30 \mathrm{~mm}$, and 120 $\mathrm{mm}$ respectively. Note that gears $B$ and $C$ are fixed connected to the same shaft [28]."

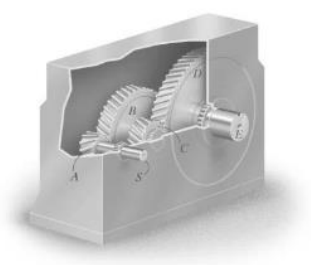

Fig. 6 Speed reducer [28].

\subsubsection{Actual Solution}

Angular velocity,

$$
\begin{aligned}
\omega & =\int_{0}^{t} k e^{b t} d t=\int_{0}^{t} 0.4 e^{t} d t=\left[0.4 e^{t}\right]_{0}^{2} \\
& =0.15972640247327 \mathrm{rad} / \mathrm{s}
\end{aligned}
$$

\subsubsection{Engineering Application}

The problem described and its solution are utilized in a variety of industries and machines. Factory automation, packaging machines, industrial machines, food processing machines, car production machines, machine tool industry, material handling, printing machines, automatic cutting/welding machines, machines for medical/cosmetic fields, construction machines, wood/glass processing machines, agricultural machinery, material processing machines, and so on are among the required application fields.

\section{Results and Discussion}

The engineering integral equations were solved and the actual values were determined using Maple program. For determining the most accurate method, we followed some definite steps. First, we found the numerical integration solutions of the four methods by using Python 3.8. We considered the intervals from 6 to 500. The detail of the comparison of various methods for the periodicity and accuracy is summarized in Table 1. The data for the graphs were constructed using Pandas. Two graphs were exhibited- one containing a maximum number of values and the other containing values from the selected zone. The graphs were constructed using Matplotlib.

\begin{tabular}{|c|c|c|c|c|c|}
\hline \multirow{2}{*}{ Problem Name } & \multirow{2}{*}{$\begin{array}{l}\text { Number of } \\
\text { intervals to } \\
\text { start forming } \\
\text { the most } \\
\text { accurate } \\
\text { method }\end{array}$} & \multicolumn{4}{|c|}{ Number of intervals for the most accurate method (when $n=1,2,3, \ldots$ ) } \\
\hline & & $\begin{array}{l}\text { Weddle's } \\
\text { method }\end{array}$ & $\begin{array}{l}\text { Simpson's } 1 / 3 \\
\text { method }\end{array}$ & $\begin{array}{l}\text { Simpson's } 3 / 8 \\
\text { method }\end{array}$ & $\begin{array}{l}\text { Trapezoidal } \\
\text { method }\end{array}$ \\
\hline Spring & 6 & $6 n$ & $6 n+2$ or $6 n+4$ & $6 n+3$ & $6 n+1$ or $6 n+5$ \\
\hline Capacitor & 204 & $6 n$ & $6 n+2$ or $6 n+4$ & $\begin{array}{c}6 n+1 \text { or } 6 n+3 \text { or } \\
6 n+5\end{array}$ & No interval \\
\hline $\begin{array}{l}\text { Volume flow rate of turbulent } \\
\text { flow }\end{array}$ & 24 & $\begin{array}{c}6 n \text { or } 6 n+2 \text { or } \\
6 n+4\end{array}$ & No interval & $6 n+3$ & $6 n+1$ or $6 n+5$ \\
\hline Wind force & 30 & $6 n$ & $6 n+2$ or $6 n+4$ & $\begin{array}{c}6 n+1 \text { or } 6 n+3 \text { or } \\
6 n+5\end{array}$ & No interval \\
\hline Total momentum flow & 18 & $\begin{array}{c}6 n \text { or } 6 n+2 \text { or } \\
6 n+4\end{array}$ & No interval & $6 n+3$ & $6 n+1$ or $6 n+5$ \\
\hline Water force on a vertical plate & 54 & $6 n$ & $6 n+2$ or $6 n+4$ & $6 n+3$ & $6 n+1$ or $6 n+5$ \\
\hline Surface tension & 6 & $6 n$ & $6 n+2$ or $6 n+4$ & $6 n+3$ & $6 n+1$ or $6 n+5$ \\
\hline Force on a sailboat mast & 18 & $6 n$ & $6 n+2$ or $6 n+4$ & $6 n+3$ & $6 n+1$ or $6 n+5$ \\
\hline Mass flow rate & 6 & $6 n$ & $6 n+2$ or $6 n+4$ & $6 n+3$ & $6 n+1$ or $6 n+5$ \\
\hline Rocket height & 6 & $6 n$ & $6 n+2$ or $6 n+4$ & $6 n+3$ & $6 n+1$ or $6 n+5$ \\
\hline Paratroopers & 42 & $6 n$ & $6 n+2$ or $6 n+4$ & $6 n+3$ & $6 n+1$ or $6 n+5$ \\
\hline Horizontal deflection & 6 & $6 n$ & $6 n+2$ or $6 n+4$ & $6 n+3$ & $6 n+1$ or $6 n+5$ \\
\hline $\begin{array}{l}\text { Block-type hand brake (moment } \\
\text { of frictional forces) }\end{array}$ & 6 & $6 n$ & $6 n+2$ or $6 n+4$ & $6 n+3$ & $6 n+1$ or $6 n+5$ \\
\hline $\begin{array}{l}\text { Block-type hand brake (moment } \\
\text { of normal forces) }\end{array}$ & 6 & $6 n$ & $6 n+2$ or $6 n+4$ & $6 n+3$ & $6 n+1$ or $6 n+5$ \\
\hline Speed of block & 6 & $6 n$ & $6 n+2$ or $6 n+4$ & $6 n+3$ & $6 n+1$ or $6 n+5$ \\
\hline Rocket velocity & 6 & $6 n$ & $6 n+2$ or $6 n+4$ & $6 n+3$ & $6 n+1$ or $6 n+5$ \\
\hline Gear box & 6 & $6 n$ & $6 n+2$ or $6 n+4$ & $6 n+3$ & $6 n+1$ or $6 n+5$ \\
\hline
\end{tabular}

Table 1 Comparison of various methods for the periodicity and accuracy. 
In the spring problem, the sequence of the most accurate numerical integration method has started forming when the number of intervals was 6 . When the number of intervals was $6 n$ (where $n=1,2,3,4 \ldots .$. ), Weddle's method was found to be the most accurate. But when the number of intervals changed to $6 n$ +2 or $6 n+4$, the most accurate method was Simpson's 1/3. Simpson's $3 / 8$ method was found to be the most accurate when the number of intervals was $6 n+3$. For a number of intervals equal to $6 n+1$ or $6 n+5$, Trapezoidal method became the most accurate. The visual proof of the aforementioned sentences is shown in Fig. 7.

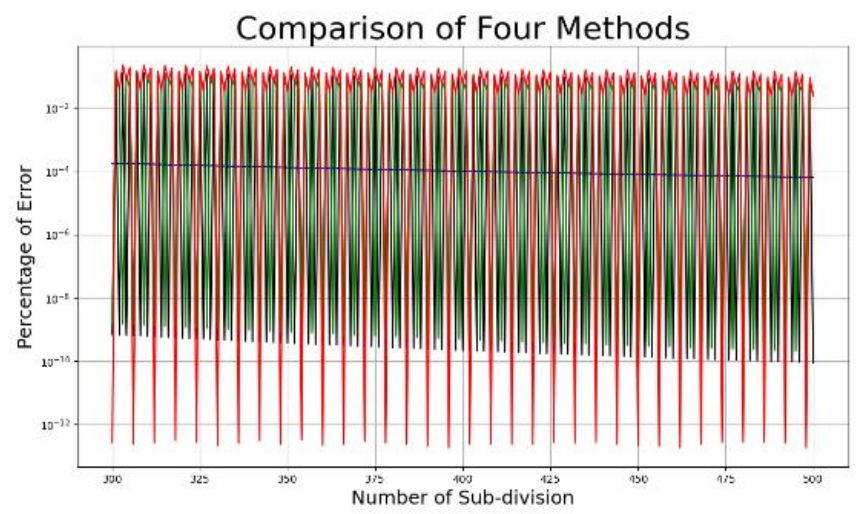

(a)

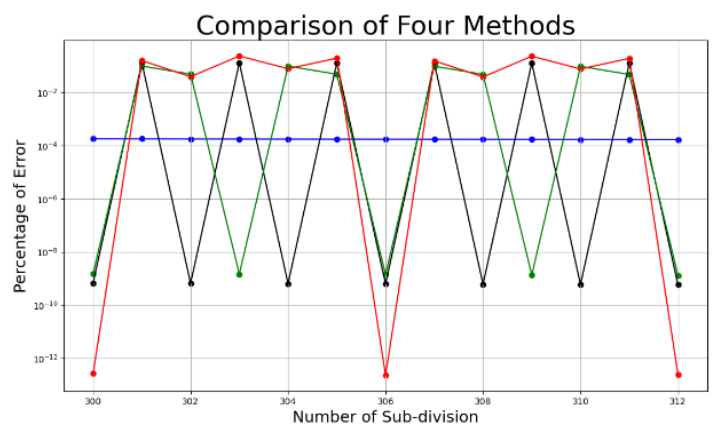

(b)

Fig. 7 Graph of Percentage of error vs. Number of Sub-division for spring problem (a) Whole (b) Magnified

In the capacitor problem, the sequence of the most accurate numerical integration method has started forming when the number of intervals was 204. When the number of intervals was $6 n$ (where $n=34,35,36,37 \ldots .$. ), Weddle's method was found to be the most accurate. But when the number of intervals changed to $6 n+2$ or $6 n+4$, Simpson's $1 / 3$ method was found to be the most accurate. Simpson's 3/8 method was found to be the most accurate when the number of intervals was $6 n+1$ or $6 n+3$ or $6 n$ +5 . There is no number of intervals for which Trapezoidal method was found to be the most accurate. The visual proof of the aforementioned sentences is shown in Fig. 8.

In the volume flow rate of the turbulent flow problem, the sequence of the most accurate numerical integration method has started forming when the number of intervals was 24 . When the number of intervals was $6 n$ or $6 n+2$ or $6 n+4$ (where $n=4,5$, $6,7 \ldots .$.$) , Weddle's method was found to be the most accurate.$ There is no number of intervals for which Simpson's 1/3 method was found to be the most accurate. But when the number of intervals changed to $6 n+3$, Simpson's $3 / 8$ method was found to be the most accurate. The trapezoidal method was found to be the most accurate when a number of intervals switched to $6 n+1$ or $6 n+5$. The visual proof of the aforementioned sentences is shown in Fig. 9.

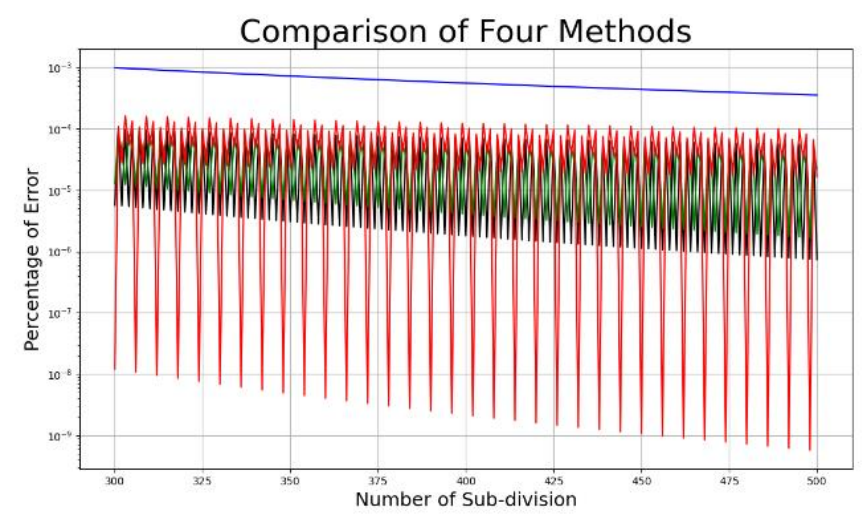

(a)
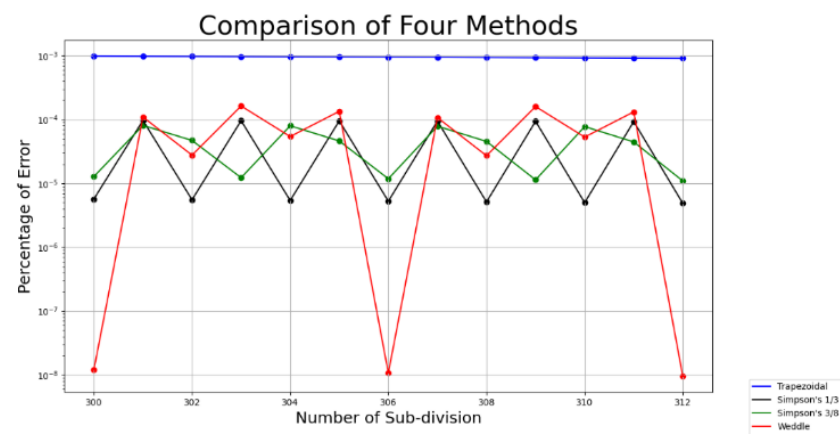

(b)

Fig. 8 Graph of Percentage of error vs. Number of Sub-division for capacitor problem (a) Whole (b) Magnified

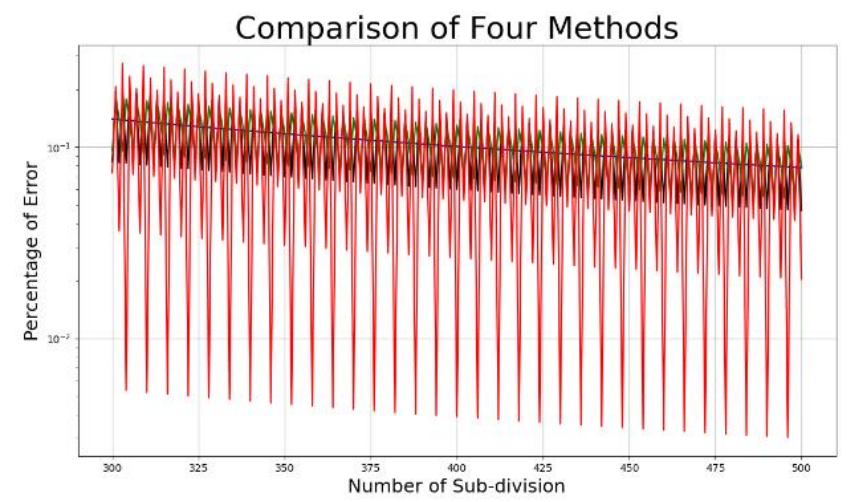

(a)
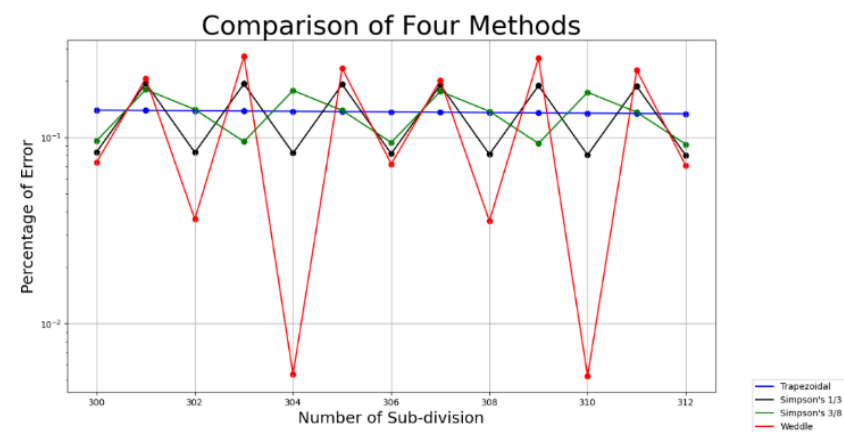

(b)

Fig. 9 Graph of Percentage of error vs. Number of Sub-division for volume flow rate of turbulent flow problem (a) Whole (b) Magnified 
In the wind force problem, the sequence of the most accurate numerical integration method has started forming when the number of intervals was 30 . When the number of intervals was $6 n$ (where $n=5,6,7,8 \ldots$. ), Weddle's method was found to be the most accurate. But when the number of intervals changed to $6 n+2$ or $6 n+4$, Simpson's $1 / 3$ method was found to be the most accurate. Simpson's $3 / 8$ method was found to be the most accurate when the number of intervals was $6 n+1$ or $6 n+3$ or $6 n$ +5 . There is no number of intervals for which the Trapezoidal method was found to be the most accurate. The visual proof of the aforementioned sentences is shown in Fig. 10.

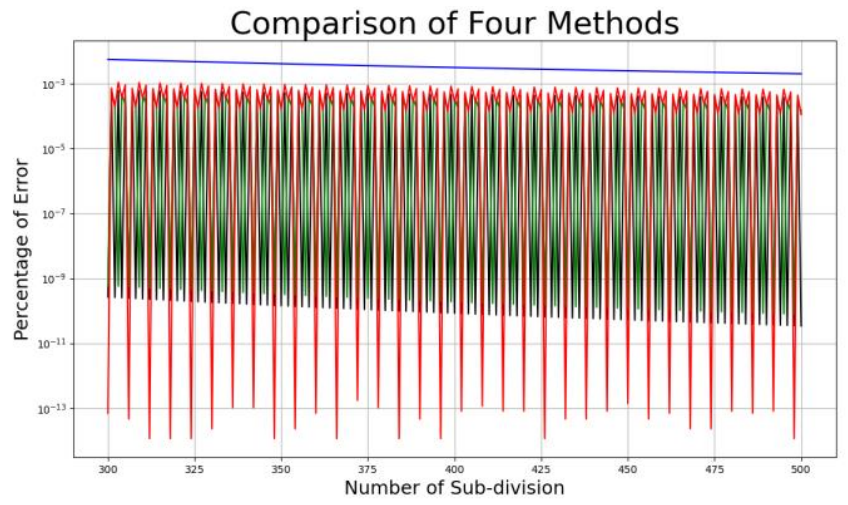

(a)
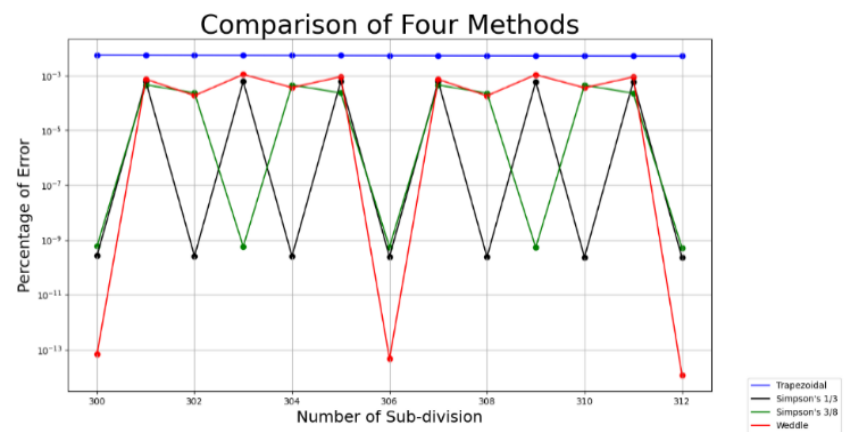

(b)

Fig. 10 Graph of Percentage of error vs. Number of Subdivision for wind force problem (a) Whole (b) Magnified

In the total momentum flow problem, the sequence of the most accurate numerical integration method has started forming when the number of intervals was 18 . When the number of intervals was $6 n$ or $6 n+2$ or $6 n+4$ (where $n=3,4,5,6 \ldots$. ), Weddle's method was found to be the most accurate. There is no number of intervals for which Simpson's 1/3 method was found to be the most accurate. But when the number of intervals changed to $6 n+3$, Simpson's $3 / 8$ method was found to be the most accurate. The trapezoidal method was found to be the most accurate when a number of intervals switched to $6 n+1$ or $6 n+$ 5 . The visual proof of the aforementioned sentences is shown in Fig. 11.

In the water force on a vertical plate problem, the sequence of the most accurate numerical integration method has started forming when the number of intervals was 54 . When the number of intervals was $6 \mathrm{n}$ (where $\mathrm{n}=9,10,11,12 \ldots .$. ), Weddle's method was found to be the most accurate. But when the number of intervals changed to $6 n+2$ or $6 n+4$, Simpson's $1 / 3$ method was found to be the most accurate. Simpson's $3 / 8$ method was found to be the most accurate when the number of intervals was $6 n+3$. The trapezoidal method was found to be the most accurate when a number of intervals switched to $6 n+1$ or $6 n+5$. The visual proof of the aforementioned sentences is shown in Fig. 12.

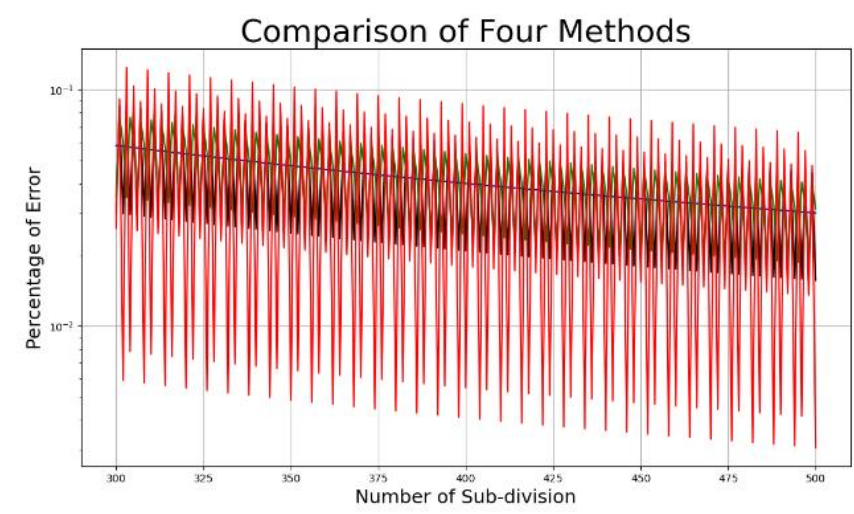

(a)

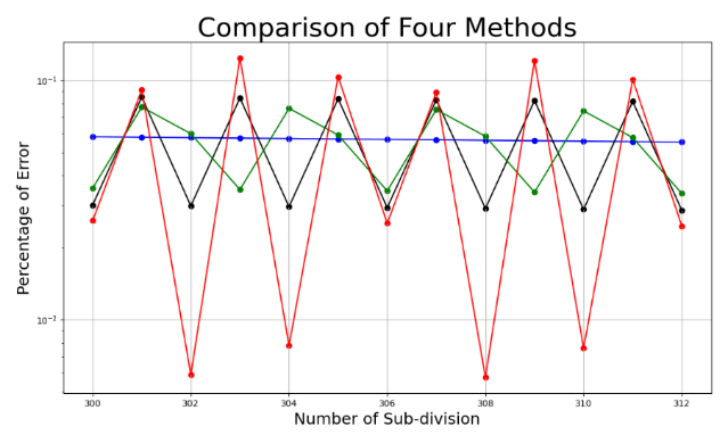

(b)

Fig. 11 Graph of Percentage of error vs. Number of Subdivision for total momentum flow problem (a) Whole (b) Magnified

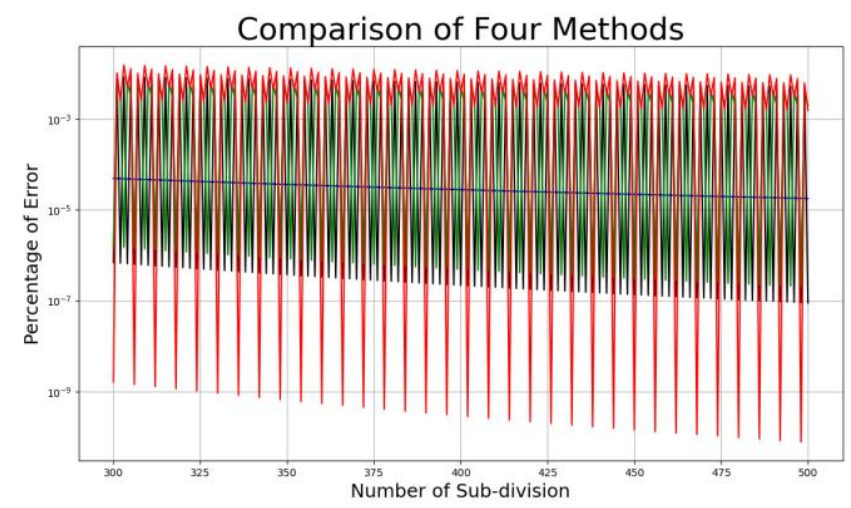

(a)

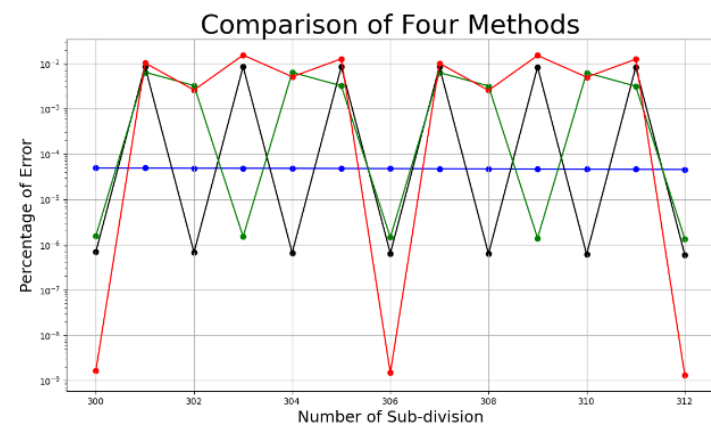

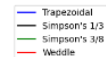

(b)

Fig. 12 Graph of Percentage of error vs. Number of Subdivision for water force on a vertical plate problem (a) Whole (b) Magnified 
In the surface tension problem, the sequence of the most accurate numerical integration method has started forming when the number of intervals was 6 . When the number of intervals was $6 n$ (where $n=1,2,3,4 \ldots$. ), Weddle's method was found to be the most accurate. But when the number of intervals changed to $6 n+2$ or $6 n+4$, Simpson's $1 / 3$ method was found to be the most accurate. Simpson's $3 / 8$ method was found to be the most accurate when the number of intervals was $6 n+3$. The trapezoidal method was found to be the most accurate when a number of intervals switched to $6 n+1$ or $6 n+5$. The visual proof of the aforementioned sentences is shown in Fig. 13.

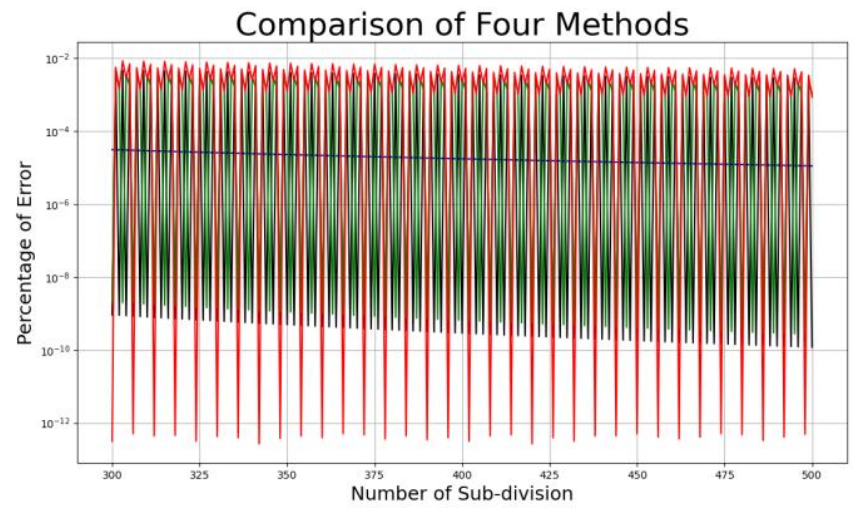

(a)
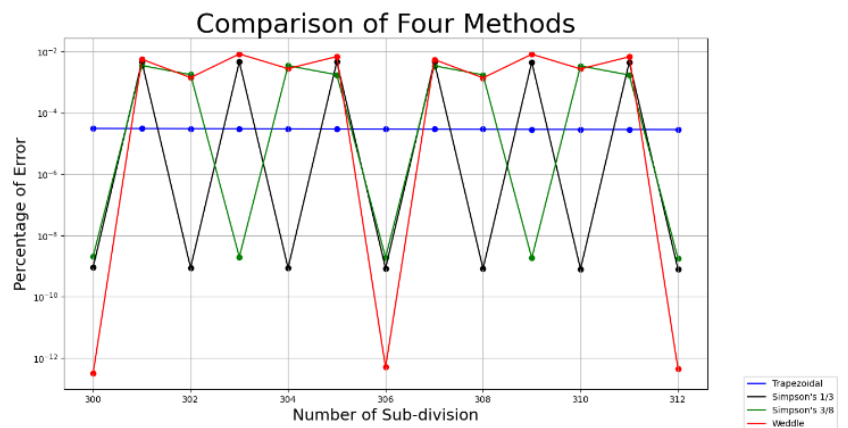

(b)

Fig. 13 Graph of Percentage of error vs. Number of Subdivision for surface tension problem (a) Whole (b) Magnified

In the force on a sailboat mast problem, the sequence of the most accurate numerical integration method has started forming when the number of intervals was 18 . When the number of intervals was $6 n$ (where $n=3,4,5,6 \ldots .$. ), Weddle's method was found to be the most accurate. But when the number of intervals changed to $6 n+2$ or $6 n+4$, Simpson's $1 / 3$ method was found to be the most accurate. Simpson's 3/8 method was found to be the most accurate when the number of intervals was $6 n+3$. The trapezoidal method was found to be the most accurate when a number of intervals switched to $6 n+1$ or $6 n+5$. The visual proof of the aforementioned sentences is shown in Fig. 14.

In the mass flow rate problem, the sequence of the most accurate numerical integration method has started forming when the number of intervals was 6 . When the number of intervals was $6 n$ (where $n=1,2,3,4 \ldots$. ), Weddle's method was found to be the most accurate. But when the number of intervals changed to $6 n+2$ or $6 n+4$, Simpson's $1 / 3$ method was found to be the most accurate. Simpson's $3 / 8$ method was found to be the most accurate when the number of intervals was $6 n+3$. The trapezoidal method was found to be the most accurate when a number of intervals switched to $6 n+1$ or $6 n+5$. The visual proof of the aforementioned sentences is shown in Fig. 15.

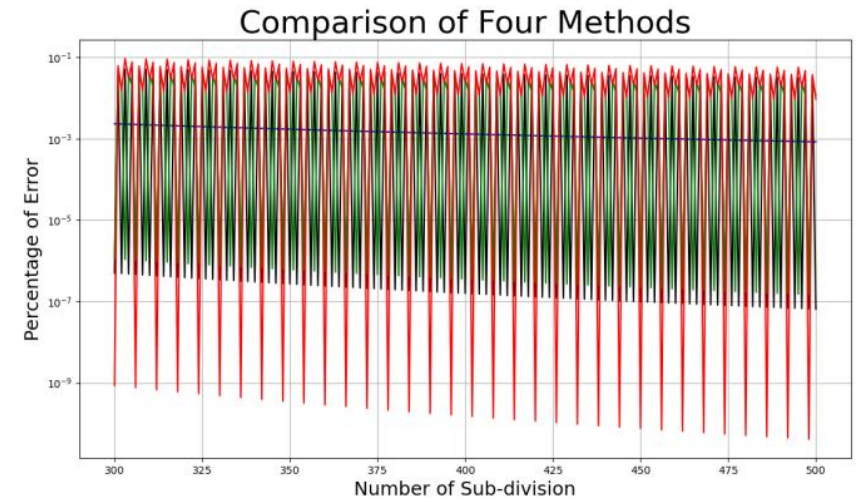

(a)

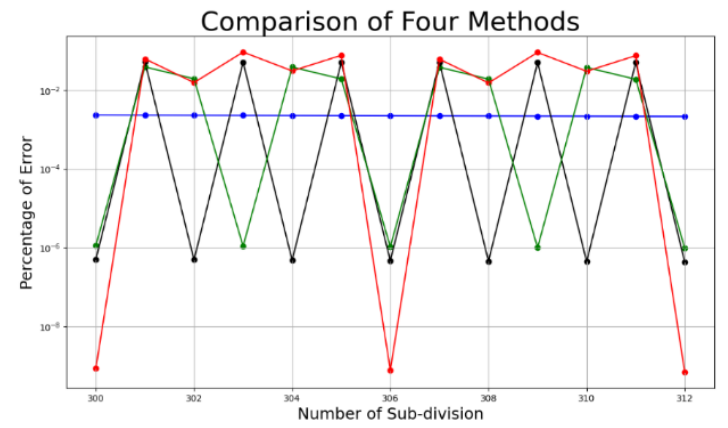

(b)

Fig. 14 Graph of Percentage of error vs. Number of Subdivision for force on a sailboat mast problem (a) Whole (b) Magnified

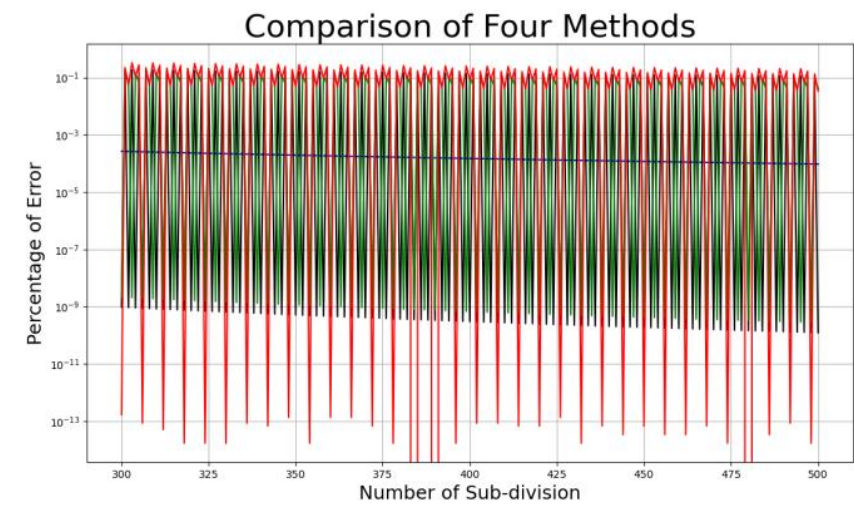

(a)
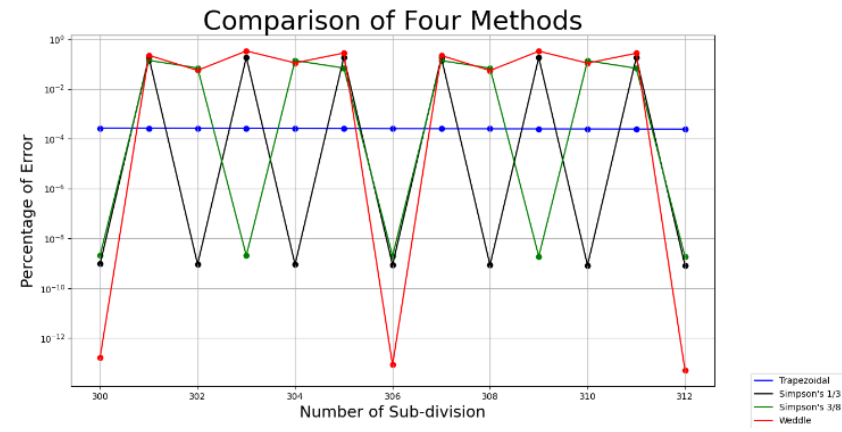

(b)

Fig. 15 Graph of Percentage of error vs. Number of Subdivision for mass flow rate problem (a) Whole (b) Magnified

In the rocket height problem, the sequence of the most accurate numerical integration method has started forming when 
the number of intervals was 6 . When the number of intervals was $6 n$ (where $n=1,2,3,4 \ldots .$. ), Weddle's method was found to be the most accurate. But when the number of intervals changed to $6 n+2$ or $6 n+4$, Simpson's $1 / 3$ method was found to be the most accurate. Simpson's $3 / 8$ method was found to be the most accurate when the number of intervals was $6 n+3$. The trapezoidal method was found to be the most accurate when a number of intervals switched to $6 n+1$ or $6 n+5$. The visual proof of the aforementioned sentences is shown in Fig. 16.

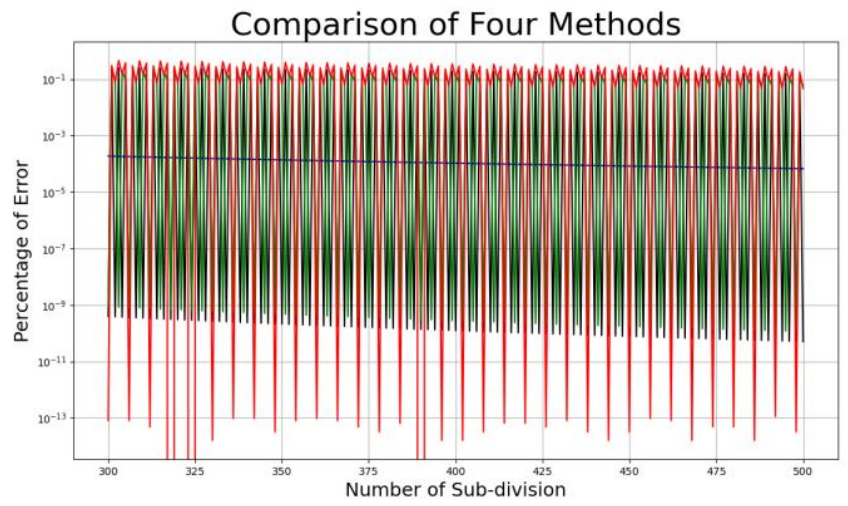

(a)

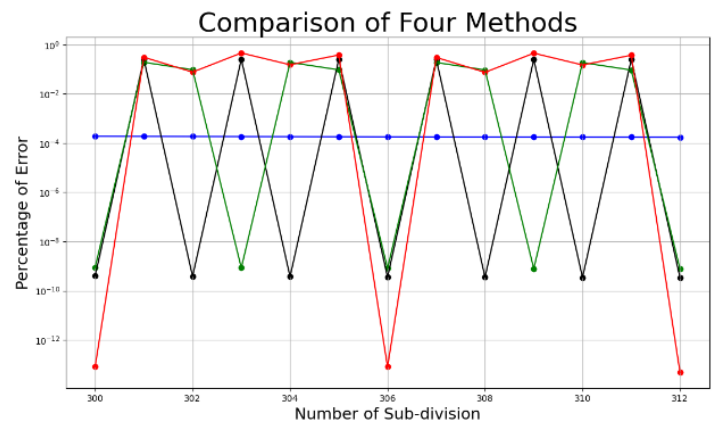

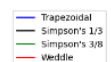

(b)

Fig. 16 Graph of Percentage of error vs. Number of Subdivision for rocket height problem (a) Whole (b) Magnified

In the paratroopers problem, the sequence of the most accurate numerical integration method has started forming when the number of intervals was 42 . When the number of intervals was $6 n$ (where $n=7,8,9,10 \ldots .$. ), Weddle's method was found to be the most accurate. But when the number of intervals changed to $6 n+2$ or $6 n+4$, Simpson's $1 / 3$ method was found to be the most accurate. Simpson's 3/8 method was found to be the most accurate when the number of intervals was $6 n+3$. The trapezoidal method was found to be the most accurate when a number of intervals switched to $6 n+1$ or $6 n+5$. The visual proof of the aforementioned sentences is shown in Fig. 17.

In the horizontal deflection problem, the sequence of the most accurate numerical integration method has started forming when the number of intervals was 6 . When the number of intervals was $6 n$ (where $n=1,2,3,4 \ldots .$. ), Weddle's method was found to be the most accurate. But when the number of intervals changed to $6 n+2$ or $6 n+4$, Simpson's $1 / 3$ method was found to be the most accurate. Simpson's 3/8 method was found to be the most accurate when the number of intervals was $6 n+3$. The trapezoidal method was found to be the most accurate when a number of intervals switched to $6 n+1$ or $6 n+5$. The visual proof of the aforementioned sentences is shown in Fig. 18.

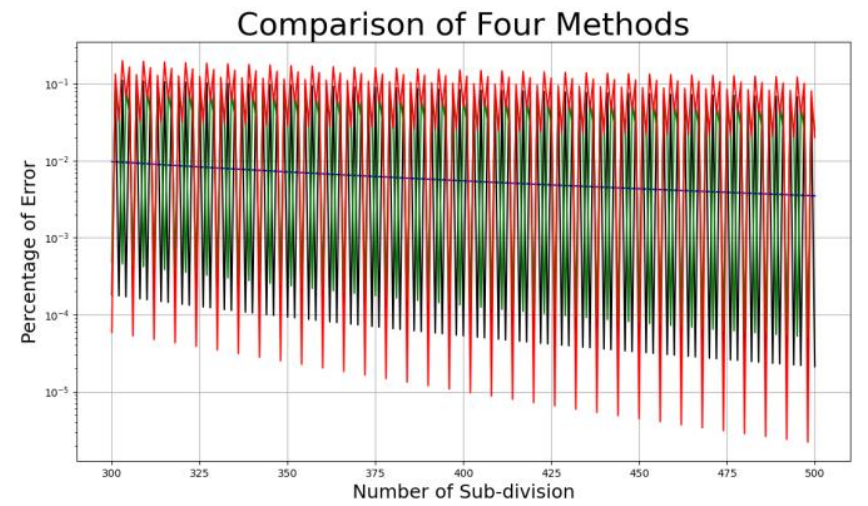

(a)
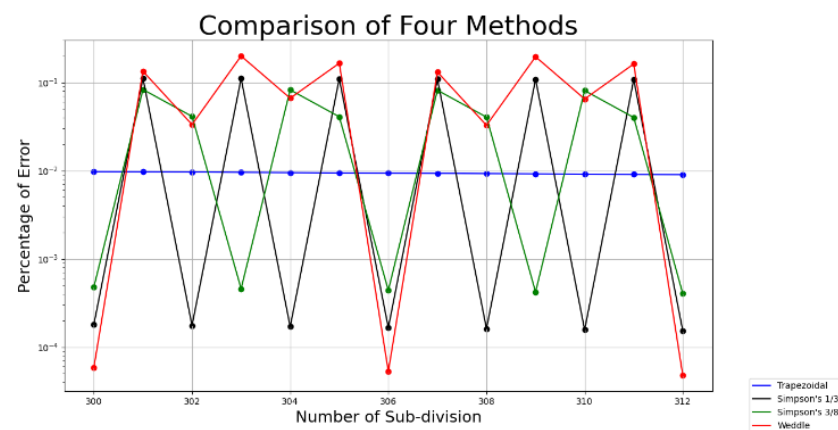

(b)

Fig. 17 Graph of Percentage of error vs. Number of Subdivision for paratroopers problem (a) Whole (b) Magnified

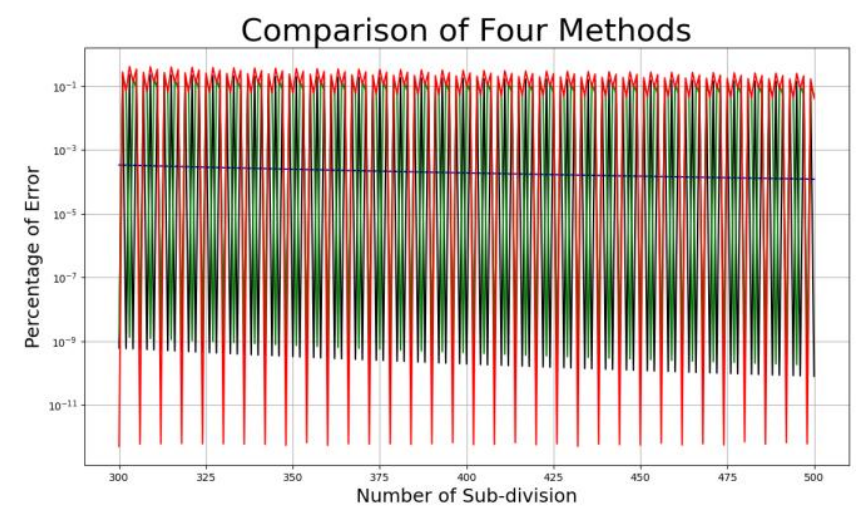

(a)
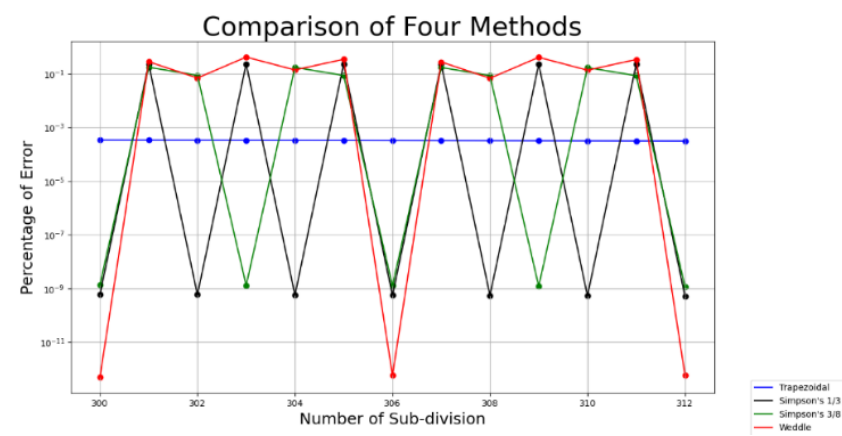

(b)

Fig. 18 Graph of Percentage of error vs. Number of Subdivision for horizontal deflection problem (a) Whole (b) Magnified 
In the block-type hand brake (moment of frictional forces) problem, the sequence of the most accurate numerical integration method has started forming when the number of intervals was 6 . When the number of intervals was $6 n$ (where $n=1,2,3,4 \ldots$. ), Weddle's method was found to be the most accurate. But when the number of intervals changed to $6 n+2$ or $6 n+4$, Simpson's $1 / 3$ method was found to be the most accurate. Simpson's $3 / 8$ method was found to be the most accurate when the number of intervals was $6 n+3$. The trapezoidal method was found to be the most accurate when a number of intervals switched to $6 n+1$ or $6 n+5$. The visual proof of the aforementioned sentences is shown in Fig. 19.

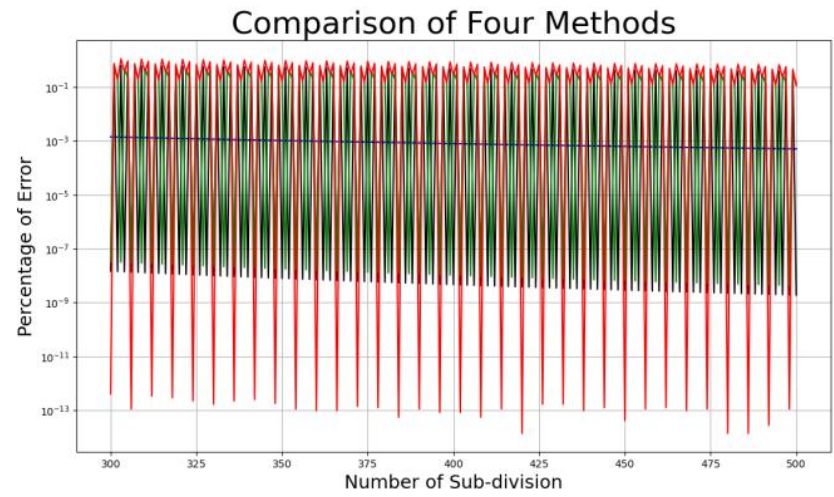

(a)
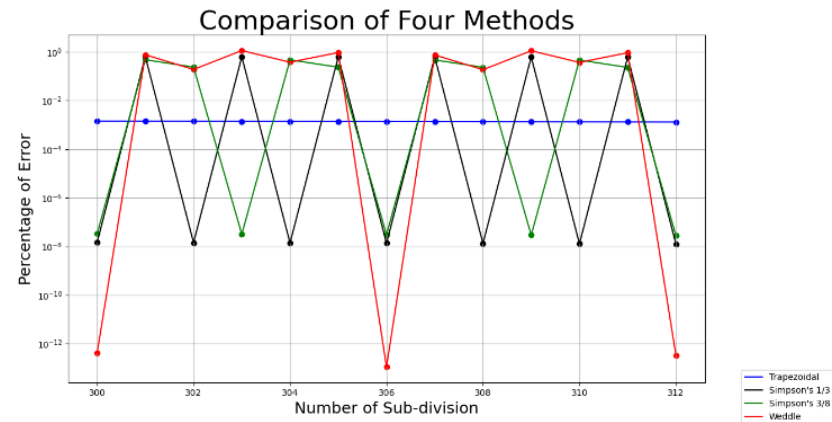

(b)

Fig. 19 Graph of Percentage of error vs. Number of Subdivision for block-type hand brake (moment of frictional forces) problem (a) Whole (b) Magnified

In the block-type hand brake (moment of normal forces) problem, the sequence of the most accurate numerical integration method has started forming when the number of intervals was 6 . When the number of intervals was $6 n$ (where $n=1,2,3,4 \ldots$. ), Weddle's method was found to be the most accurate. But when the number of intervals changed to $6 n+2$ or $6 n+4$, Simpson's $1 / 3$ method was found to be the most accurate. Simpson's 3/8 method was found to be the most accurate when the number of intervals was $6 n+3$. The trapezoidal method was found to be the most accurate when a number of intervals switched to $6 n+1$ or $6 n+5$. The visual proof of the aforementioned sentences is shown in Fig. 20.

In the speed of block problem, the sequence of the most accurate numerical integration method has started forming when the number of intervals was 6 . When the number of intervals was $6 n$ (where $n=1,2,3,4 \ldots .$. ), Weddle's method was found to be the most accurate. But when the number of intervals changed to $6 n+2$ or $6 n+4$, Simpson's $1 / 3$ method was found to be the most accurate. Simpson's 3/8 method was found to be the most accurate when the number of intervals was $6 n+3$. The trapezoidal method was found to be the most accurate when a number of intervals switched to $6 n+1$ or $6 n+5$. The visual proof of the aforementioned sentences is shown in Fig. 21.

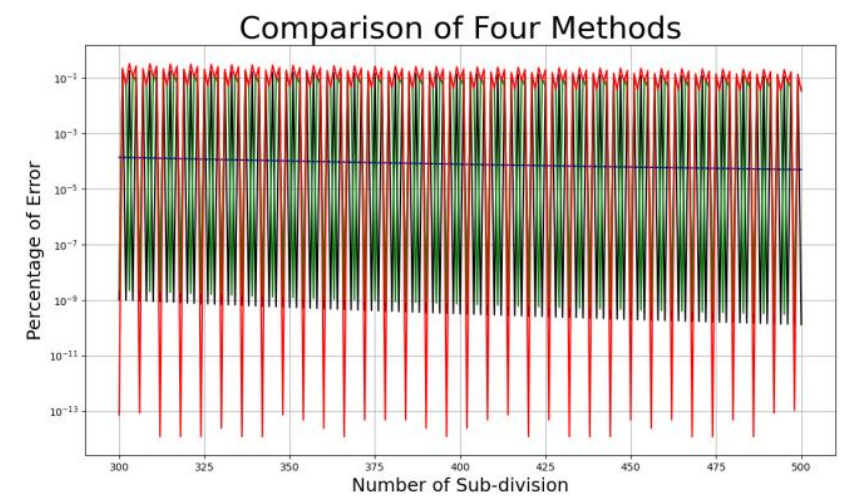

(a)

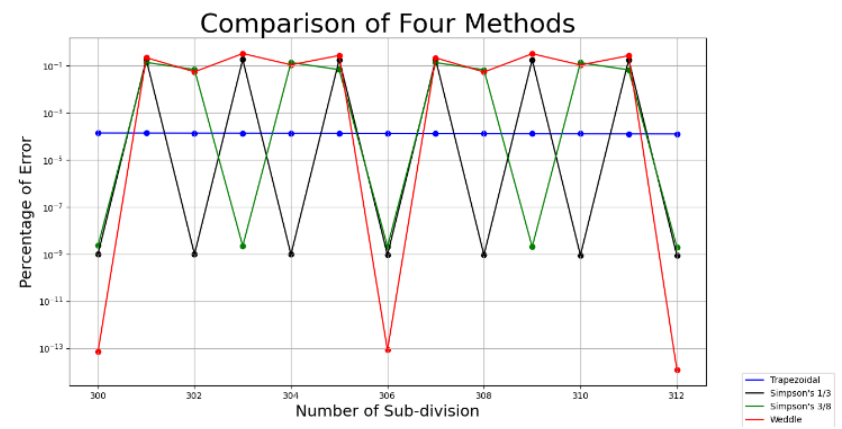

(b)

Fig. 20 Graph of Percentage of error vs. Number of Subdivision for block-type hand brake (moment of frictional forces) problem (a) Whole (b) Magnified

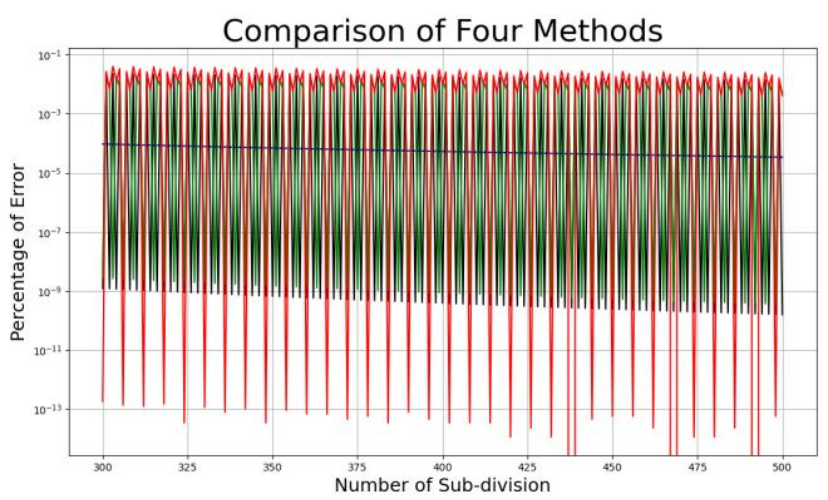

(a)

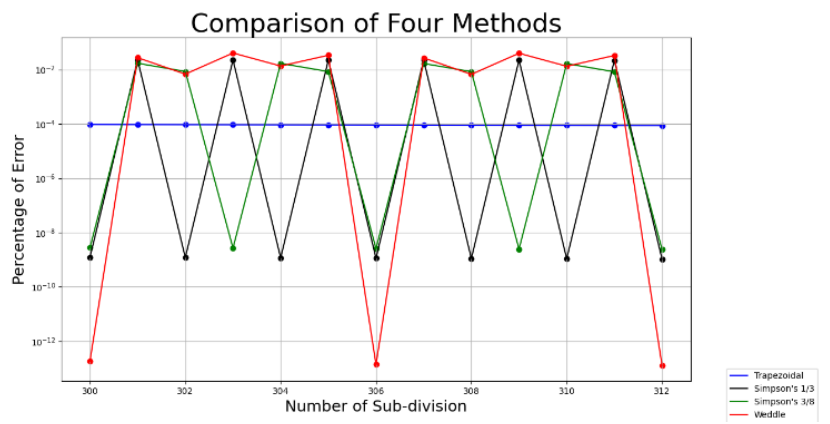

(b)

Fig. 21 Graph of Percentage of error vs. Number of Subdivision for speed of block problem (a) Whole (b) Magnified 


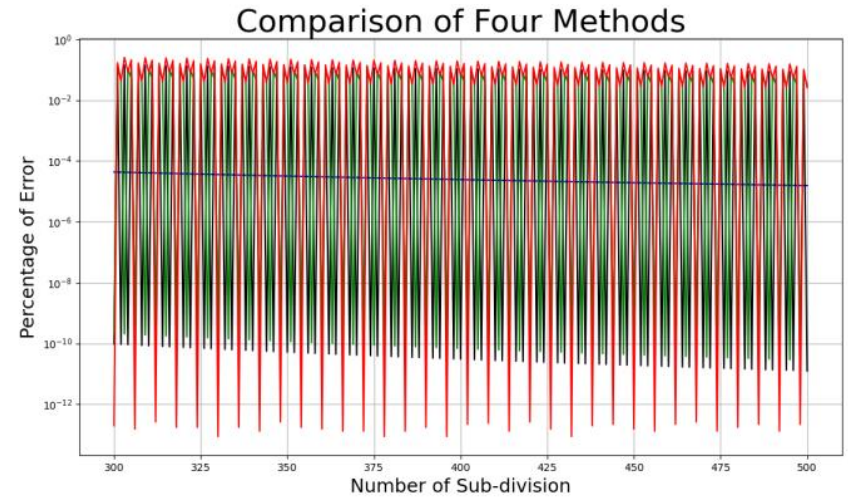

(a)

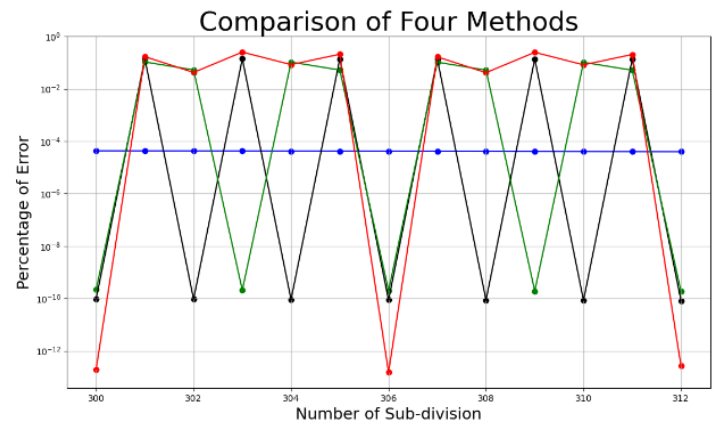

(b)

Fig. 22 Graph of Percentage of error vs. Number of Subdivision for rocket velocity problem (a) Whole (b) Magnified

In the rocket velocity problem, the sequence of the most accurate numerical integration method has started forming when the number of intervals was 6 . When the number of intervals was $6 n$ (where $n=1,2,3,4 \ldots .$. ), Weddle's method was found to be the most accurate. But when the number of intervals changed to $6 n+2$ or $6 n+4$, Simpson's $1 / 3$ method was found to be the most accurate. Simpson's $3 / 8$ method was found to be the most accurate when the number of intervals was $6 n+3$. The trapezoidal method was found to be the most accurate when a number of intervals switched to $6 n+1$ or $6 n+5$. The visual proof of the aforementioned sentences is shown in Fig. 22.

In the gear box problem, the sequence of the most accurate numerical integration method has started forming when the number of intervals was 6 . When the number of intervals was $6 n$ (where $n=1,2,3,4 \ldots$. ), Weddle's method was found to be the most accurate. But when the number of intervals changed to $6 n$ +2 or $6 n+4$, Simpson's $1 / 3$ method was found to be the most accurate. Simpson's $3 / 8$ method was found to be the most accurate when the number of intervals was $6 n+3$. The trapezoidal method was found to be the most accurate when a number of intervals switched to $6 n+1$ or $6 n+5$. The visual proof of the aforementioned sentences is shown in Fig. 23.

\section{Discussion}

The solved problems along with their sequences of accurate methods are shown below with a Sunburst Chart as shown in Fig. 24. In the chart, ' $\mathrm{W}$ '=Weddle's Rule, ' $\mathrm{T}$ '=Trapezoidal Rule, 'S1/3'= Simpson's 1/3 Rule, and 'S3/8'=Simpson's 3/8 Rule.
From Fig. 24, it is clearly visible that most of the selected engineering problems follow a common sequence of ' $\mathrm{W}-\mathrm{T}-\mathrm{S} 1 / 3$ S3/8-S1/3-T-W'. In total, 13 out of 17 problems exhibit this periodic sequence of accuracy. Also, 2 out of 17 demonstrate a sequence of ' $\mathrm{W}-\mathrm{S} 3 / 8-\mathrm{S} 1 / 3-\mathrm{S} 3 / 8-\mathrm{S} 1 / 3-\mathrm{S} 3 / 8-\mathrm{W}$ '. Interestingly, trapezoidal method is never the most accurate one for these two problems. Finally, 2 out of 17 problems show a sequence of ' $\mathrm{W}$ T-W-T-W-S3/8-W'. Simpson's $1 / 3$ is never the most accurate method for these two problems.

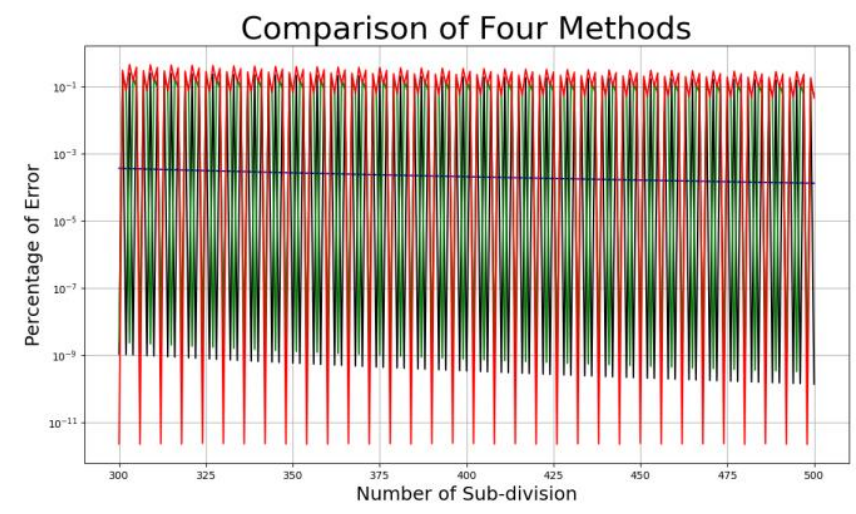

(a)
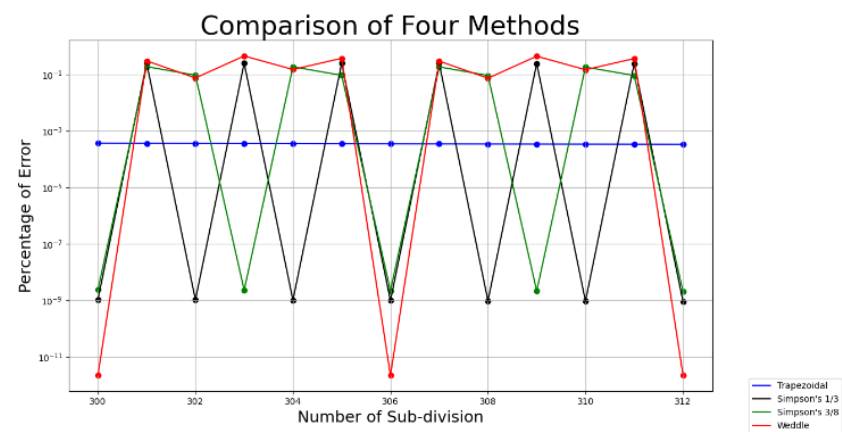

(b)

Fig. 23 Graph of Percentage of error vs. Number of Subdivision for gear box problem (a) Whole (b) Magnified

\section{Conclusion}

In our paper, some integration problems were brought together and solved by using Python 3.8. The graphs and the data for individual problems were found using Matplotlib and Pandas respectively. The problems were chosen meticulously according to their application in the different engineering fields. By analyzing the problems, it was found that there was no single numerical integration method among the Newton-Cotes methods whose accuracy was constantly maintained throughout the interval. Rather, it is seen that with the change of interval, the most accurate method changes and there is an interesting pattern of accuracy that is followed in each engineering problem. This will give insights to the scientists and engineers working in the field, on what methods they can use according to their necessary accuracy demand of their industries. This can further be extended to the other engineering applications as well so that the most accurate value within a particular interval can be known. 


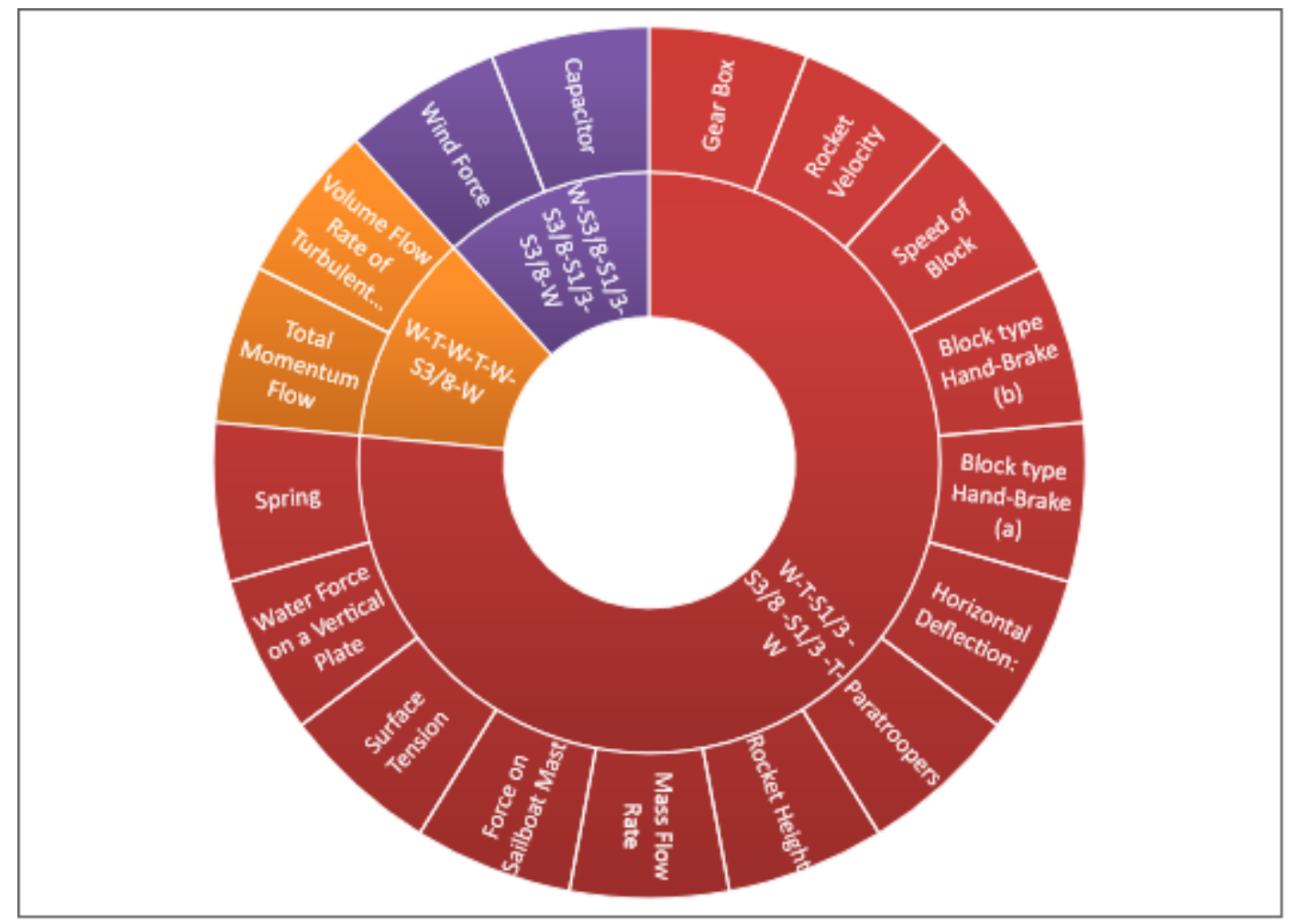

Fig. 24 Demonstration of all the engineering problems with their accuracy sequence by a Sunburst Chart

\section{References}

[1] Sastry, S.S., 2007. Introductory methods of numerical analysis. PHI Learning Pvt. Ltd..

[2] Burden, R.L., 2007. Numerical analysis, $7^{\text {th }}$ Edition, International Thomson Publishing Company.

[3] Mathews, J.H., 2000. Numerical methods for mathematics, science and engineering. Prentice-Hall International., $2^{\text {nd }}$ Edition, Prentice Hall of India Private Limited.

[4] Ausin, M.C., 2007. An introduction to quadrature and other numerical integration techniques. Encyclopedia of Statistics in Quality and reliability. Chichester, England.

[5] Smith G.K., 2004. Numerical Integration, Encyclopedia of Biostatistics. $2^{\text {nd }}$ edition, Vol-6.

[6] Sinha, R.K. and Kumar, R., 2010. Numerical method for evaluating the integrable function on a finite interval. International Journal of Engineering Science and Technology, 2(6), pp.2200-2206.

[7] Sozio, G., 2009. Numerical integration. Australian Senior Mathematics Journal, 23(1), pp.43-50.

[8] Oliver, J., 1971. The evaluation of definite integrals using highorder formulae. The Computer Journal, 14(3), pp.301-306.

[9] Natarajan, A. and Mohankumar, N., 1995. A comparison of some quadrature methods for approximating Cauchy principal value integrals. Journal of Computational Physics, 116(2), pp.365-368.

[10] Liu, D., Wu, J. and Yu, D., 2010. The superconvergence of the Newton-Cotes rule for Cauchy principal value integrals. Journal of computational and applied mathematics, 235(3), pp.696-707.

[11] Muthumalai, R.K., 2012. Some Formulae for Numerical Differentiation through Divided Difference. International Journal of Mathematical Archive, 3.

[12] Khan, M.M.U.R., Hossain, M.R. and Parvin, S., 2017. Numerical integration schemes for unequal data spacing. American Journal of Applied Mathematics, 5(2), pp.48-56.

[13] Bailey, D.H. and Borwein, J.M., 2011. High-precision numerical integration: Progress and challenges. Journal of Symbolic Computation, 46(7), pp.741-754.
[14] White, G.C. and Cooch, E.G., 2017. Population abundance estimation with heterogeneous encounter probabilities using numerical integration. The Journal of Wildlife Management, 81(2), pp.322-336.

[15] Wei, W., Zhou, B., Połap, D. and Woźniak, M., 2019. A regional adaptive variational PDE model for computed tomography image reconstruction. Pattern Recognition, 92, pp.64-81.

[16] Liang, M. and Simos, T.E., 2016. A new four stages symmetric twostep method with vanished phase-lag and its first derivative for the numerical integration of the Schrödinger equation. Journal of Mathematical Chemistry, 54(5), pp.1187-1211.

[17] Chapra, S.C., 2008. Applied numerical methods with MATLAB for engineers and scientists. McGraw-Hill Higher Education. 4th edition. ISBN-13: 978-0073397962.

[18] Fausett, L.V., 2002. Numerical methods using MathCAD. Pearson.

[19] Siauw, T. and Bayen, A., 2014. An introduction to MATLAB® programming and numerical methods for engineers. Academic Press, Elsevier, USA, 2015.

[20] Davis, P.J. and Rabinowitz, P., 2007. Methods of numerical integration, Dover Publications, Mineola, New York, 2007.

[21] Chapra, S.C. and Canale, R.P., 2010. Numerical methods for engineers, McGraw-Hill. Inc., New York.

[22] Caligaris, M., Rodríguez, G. and Laugero, L., 2015. Designing tools for numerical integration. Procedia-Social and Behavioral Sciences, 176, pp.270-275.

[23] Kiusalaas, J., 2005. Numerical methods in engineering with $M A T L A B \circledR$. Cambridge university press.

[24] Jaluria, Y., 2011. Computer methods for engineering with MATLAB applications. CRC Press.

[25] Chapra, S.C., 2008. Applied numerical methods with MATLAB for engineers and scientists (pp. 335-359). McGraw-Hill Higher Education.

[26] Hsu, T.R., 2017. Applied engineering analysis. John Wiley \& Sons.

[27] Budynas, R.G. and Nisbett, J.K., 2011. Shigley's mechanical engineering design (Vol. 9). New York: McGraw-Hill.

[28] Hibbeler, R.C., 2004. Engineering mechanics: dynamics. Pearson. 